\title{
FRONTIER RESEARCH IN ASTROPHYSICS: AN UPDATED REVIEW
}

\author{
F. Giovannelli ${ }^{1}$ and L. Sabau-Graziati ${ }^{2}$
}

\begin{abstract}
This article is a summary of the updated version of the review article "The impact of the space experiments on our knowledge of the physics of the Universe" (Giovannelli \& Sabau-Graziati 2004: GSG2004) and subsequent slow updating (Giovannelli 2013; Giovannelli \& Sabau-Graziati 2012a, 2014a,b, 2015, 2016a,b). We will go along different stages of the evolution of our Universe discussing briefly several examples of results that, in accordance with our opinion, are the pillars carrying the Bridge between the Big Bang and Biology. A significant part of these results come from great experiments in Earth or from space. Similarly, small experiments on Earth or in space have provided - and will provide - significant results. Due to the limited extension of this work and according to our knowledge, we have made a strict selection of the topics.
\end{abstract}

\section{RESUMEN}

Este artículo es un resumen de la versión actualizada del artículo de revisión "El impacto de los experimentos espaciales en nuestro conocimiento de la física del universo" (Giovannelli y Sabau-Graziati 2004: GSG2004) y de las lentas posteriores modificaciones realizadas (Giovannelli 2013; Giovannelli y Sabau-Graziati 2012a, 2014a,b, 2015, 2016a,b). Recorreremos las diferentes etapas de la evolución de nuestro Universo tratando brevemente varios ejemplos de los resultados, que, en nuestra opinión, constituyen los pilares que sostienen el puente entre el Big Bang y la Biología. Una parte significativa de estos resultados provienen de grandes experimentos realizados en Tierra o desde el espacio. De igual manera pequeños experimentos realizados en Tierra o en el espacio han proporcionado - y proporcionarán - importantes resultados. Debido a la limitada extensión de este trabajo y en función de nuestro conocimiento, hemos realizado una estricta selección de los temas tratados.

Key Words: cosmology — gamma-ray bursts — gravitational waves — X-ray binaries

\section{INTRODUCTION}

The birth of the universe and its present status constitute the two banks of a river in which the life of the universe is slowly flowing. Undoubtedly the two banks are joined by a bridge that Giovannelli (2001a) nicknamed "The Bridge between the Big Bang and Biology" that constituted the title of the workshop held in Stromboli (Aeolian Archipelago, Sicily, Italy) in 1999. The big problem is how to cross this bridge, and the main question is: what are the experimental tools for understanding the pillars of this Bridge?

In order to cross this bridge, as always when we cross a bridge, we MUST advance slowly, step by step, with continuity, because everything is smoothly linked in the "magma" of the Universe, from the infinitely small to infinitely big, as sketched in Figure 1 (Rees 1988).

${ }^{1}$ INAF - Istituto di Astrofisica e Planetologia Spaziali, Area di Ricerca di Roma-2, Via Fosso del Cavaliere, 100, I 00133 Roma, Italy (franco.giovannelli@iaps.inaf.it).

${ }^{2}$ INTA - Dpt de Cargas Utiles y Ciencias del Espacio Ctra de Ajalvir Km 4 - E 28850 Torrejón de Ardóz, Spain (sabaumd@inta.es).

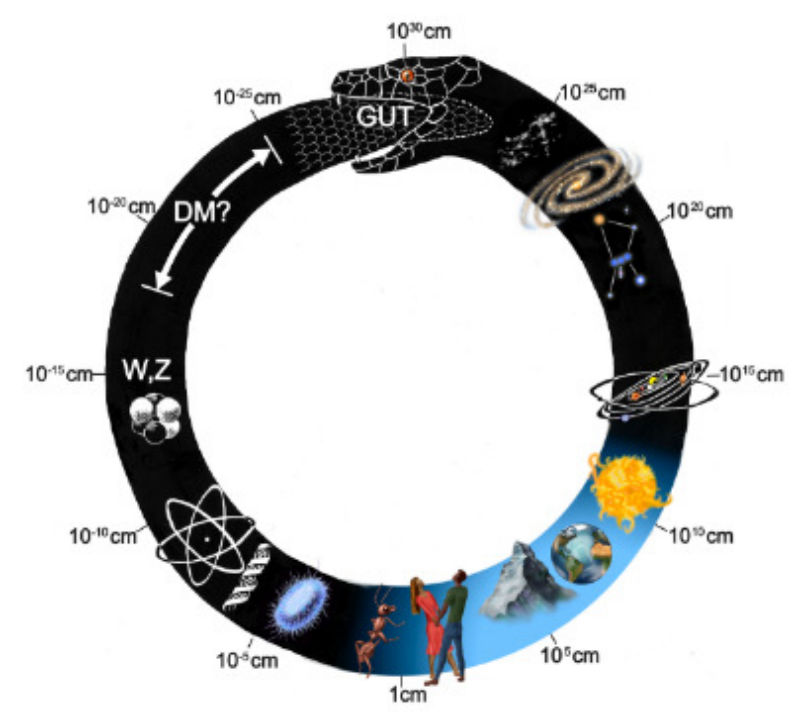

Fig. 1. From the infinitely small to infinitely big (adopted from Rees 1988).

Indeed, if we look at the Figure 2 (upper panel) - where a section of the metabolic network of a "simple" bacterium is shown - we can note 


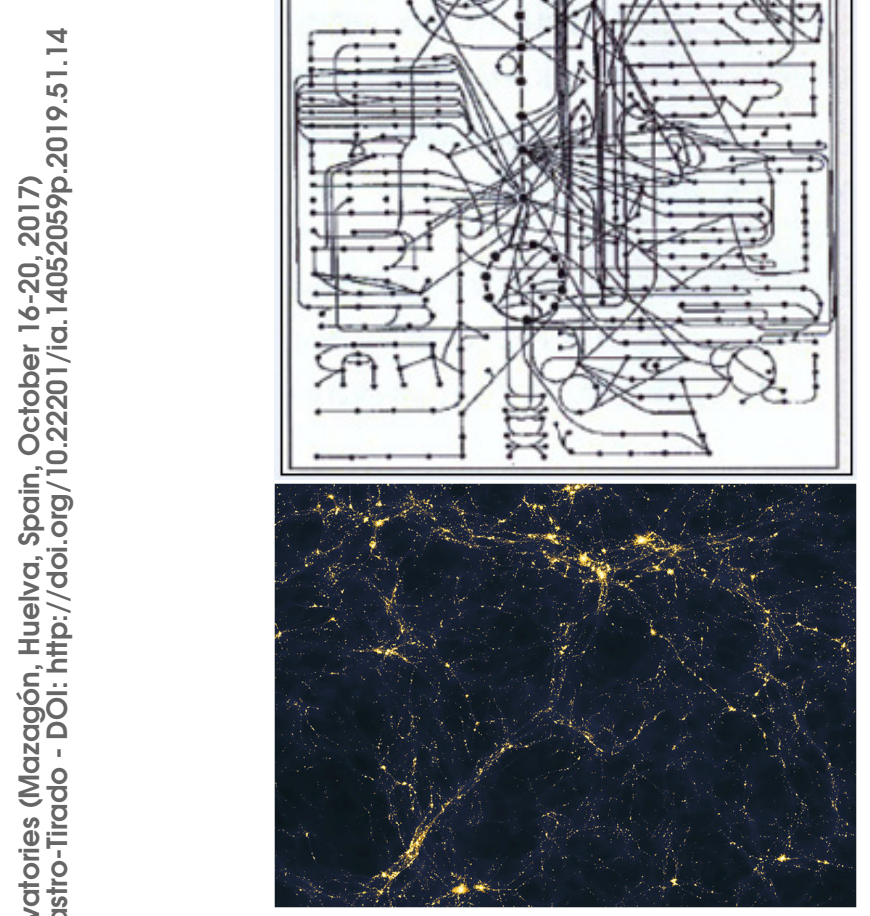

Fig. 2. Upper panel: Section of the metabolic network of a "simple" bacterium (Luisi \& Capra 2014). Lower panel: the "cosmic network" (https://it.wikipedia. org/wiki/Cosmologiadelplasma).

that each point (each chemical compound) is connected to any other point through the complexity of the network (Luisi \& Capra 2014) exactly the same occurring in the "cosmic network" where each point is connected to any other point through the complexity of the network as shown in Figure 2 (lower panel) (https://it.wikipedia.org/wiki/ Cosmologiadelplasma). The large-scale structure of the Universe, as traced by the distribution of galaxies, is now being revealed by large-volume cosmological surveys. The structure is characterized by galaxies distributed along filaments, the filaments connecting in turn to form a percolating network. Shandarin, Habib \& Heitmann (2010) objective was to quantitatively specify the underlying mechanisms that drive the formation of the cosmic network. By combining percolation-based analyses with Nbody simulations of gravitational structure formation, they elucidate how the network has its origin

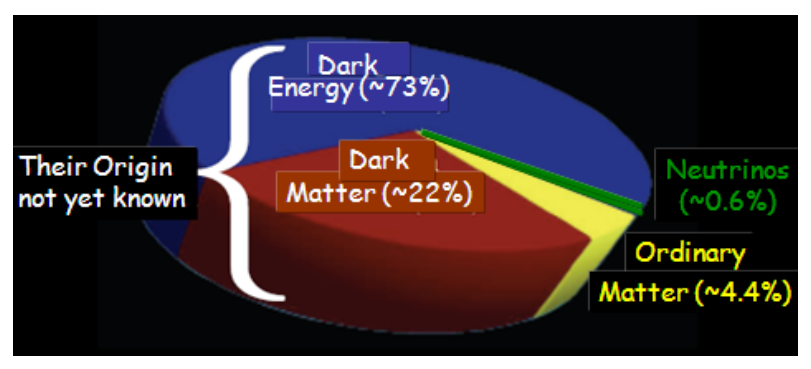

Fig. 3. The cosmic budget.

in the properties of the initial density field (nature) and how its contrast is then amplified by the nonlinear mapping induced by the gravitational instability (nurture).

As Albert Einstein declared, we can't solve problems by using the same kind of thinking we used when we created them. We can add something more, by using wisdom: we can attach each kind of problem in a way as general as possible, and in any case it is necessary to go on without blinkers.

A fundamental question naturally arises: what is now the situation about our knowledge of the Universe? The answer is discouraging: we know a very small part of it, and not very well.

Indeed, Figure 3 shows schematically such a situation. We can really discuss only about $\sim 5 \%$ of the content of the Universe. The remaining $\sim 95 \%$ is almost completely unknown. However, the recent fundamental progress in gravitational astronomy could open an incredible source of information about this "unknown" content.

In this paper we will briefly discuss the main pillars of this bridge by using the huge amount of experimental data coming from "Active Physics Experiments (APEs)" and from "Passive Physics Experiments (PPEs)", both ground- and space-based. The APEs try to reproduce in the laboratory the physical conditions of our Universe at the beginning of its life and later, while the PPEs try to observe our Universe after the epoch of recombination, when the Cosmic Microwave Background (CMB) gives witness of the conditions of the primeval Universe, and later - after the epoch of reionization, when the first stars appear - for providing information about the formation of galaxies, "active" and "normal", quasars (QSOs), and all the processes giving rise to Gamma Ray Bursts (GRBs), stellar evolution and Supernovae explosions. These latter phenomena are responsible of the injection of heavy elements in the interstellar medium, the condition necessary for the formation of rocky planets, and thus for the flowering of life. 
Before going along the different stages of the evolution of our Universe crossing the Bridge between the Big Bang and Biology we need to review a fundamental antecedent: nuclear reactions in stars.

\section{NUCLEAR REACTIONS IN STARS}

If we have not experimental information about the cross sections of nuclear reactions occurring in the stars it is hard to describe stellar evolution correctly.

The knowledge of the cross-sections of nuclear reactions occurring in stars appears as one of the most crucial points of all astroparticle physics. Direct measurements of the cross sections of the ${ }^{3} \mathrm{He}\left({ }^{4} \mathrm{He}, \gamma\right){ }^{7} \mathrm{Be}$ and ${ }^{7} \mathrm{Be}(\mathrm{p}, \gamma){ }^{8} \mathrm{Be}$ reactions of the $p p$ chain and ${ }^{14} \mathrm{~N}(\mathrm{p}, \gamma){ }^{15} \mathrm{O}$ reaction of the CNO-cycle allow substantial improvement in our knowledge on stellar evolution.

Wolschin (2003) published a very interesting paper about the history of the " Thermonuclear Processes in Stars and Stellar Neutrinos".

An impressive review about nuclear reactions has been published by Adelberger et al. (2011). They summarize and critically evaluate the available data on nuclear fusion cross sections important to energy generation in the Sun and other hydrogen-burning stars and to solar neutrino production. Recommended values and uncertainties are provided for key cross sections, and a recommended spectrum is given for ${ }^{8} \mathrm{~B}$ solar neutrinos. They also discuss opportunities for further increasing the precision of key rates, including new facilities, new experimental techniques, and improvements in theory. This review, which summarizes the conclusions of a workshop held in January 2009, is intended as a 10-year update and supplement to the reviews by Adelberger et al. (1998).

At the moment the LUNA (Laboratory for Underground Nuclear Astrophysics) is devoted to measuring nuclear cross sections relevant in astroparticle physics. It is the most valuable experiment running underground in the Gran Sasso Laboratory of the INFN.

The LUNA collaboration has already measured with good accuracy the key reactions $\mathrm{D}(\mathrm{p}, \gamma)^{3} \mathrm{He}$, ${ }^{3} \mathrm{He}(\mathrm{D}, \mathrm{p}){ }^{4} \mathrm{He}$ and ${ }^{3} \mathrm{He}\left({ }^{4} \mathrm{He}, \gamma\right){ }^{7} \mathrm{Be}$. These measurements substantially reduce the theoretical uncertainty of $\mathrm{D},{ }^{3} \mathrm{He},{ }^{7} \mathrm{Li}$ abundances. The $\mathrm{D}\left({ }^{4} \mathrm{He}, \gamma\right){ }^{6} \mathrm{Li}$ cross section - which is the key reaction for the determination of the primordial abundance of ${ }^{6} \mathrm{Li}$ - has been measured (e.g. Gustavino 2007, 2009, 2011, 2012, 2013), as well as that of ${ }^{2} \mathrm{H}(\alpha, \gamma){ }^{6} \mathrm{Li}$ (Anders et al. 2013), and ${ }^{2} \mathrm{H}(\alpha, \gamma){ }^{6} \mathrm{Li}$ (Anders et al. 2014).
Other reactions fundamental for a better knowledge of stellar evolution have been studied by the LUNA experiment: e.g. ${ }^{17} \mathrm{O}(\mathrm{p}, \gamma){ }^{18} \mathrm{~F}$ (Scott et al. 2012); ${ }^{25} \mathrm{Mg}(\mathrm{p}, \gamma){ }^{26} \mathrm{Al}$ (Strieder et al. 2012) ${ }^{25} \mathrm{Mg}(\mathrm{p}, \gamma){ }^{26} \mathrm{Al}$ (Straniero et al. 2013); ${ }^{17} \mathrm{O}(\mathrm{p}, \gamma){ }^{18} \mathrm{~F}$ (Di Leva et al. 2014).

A general data base for Experimental Nuclear Reaction Data (EXFOR) can be found in: https: //www-nds.iaea.org/exfor/exfor.htm.

\section{THE TOOLS FOR EXPLORING THE UNIVERSE}

The Universe manifests not only through electromagnetic radiation but also through astroparticles, including neutrinos, and recently through gravitational waves. Therefore, multifrequency observations, possibly simultaneous, are fundamental in photonic astrophysics, particle astrophysics, and gravitational wave astrophysics.

Thus it is evident the necessity of many kinds of experiments in different frequency regions. Upstream of this we have to distinguish two great classes of experiments: big experiments and small experiments.

\subsection{Big experiments}

Several examples of big experiments are:

a) The Astrometry Mission GAIA (Rix \& Bovy 2013) is making the largest, most precise threedimensional map of our Galaxy by surveying more than a thousand million stars. GAIA will monitor each of its target stars about 70 times over a fiveyear period. It will precisely chart their positions, distances, movements, and changes in brightness. It is expected to discover hundreds of thousands of new celestial objects, such as extra-solar planets and brown dwarfs, and observe hundreds of thousands of asteroids within our own Solar System. The mission will also study about 500,000 distant quasars and will provide stringent new tests of Albert Einstein's General Theory of Relativity.

GAIA is providing strong impact on stellar evolution and in calibrating the energy released by cosmic sources.

b) The European Extremely Large Telescope (E-ELT) is a revolutionary scientific project for a $40 \mathrm{~m}$-class telescope that will allow us to address many of the most pressing unsolved questions about our Universe.

The E-ELT will be the largest optical/nearinfrared telescope in the world and will gather 13 times more light than the largest optical telescopes existing today. The E-ELT will be able to correct for 
the atmospheric distortions (i.e., fully adaptive and diffraction-limited) from the start, providing images 16 times sharper than those from the Hubble Space Telescope. The E-ELT will vastly advance astrophysical knowledge by enabling detailed studies of planets around other stars, the first galaxies in the Universe, super-massive black holes, and the nature of the Universe's dark sector (Gilmozzi \& Spyromilio 2007).

The final approval of E-ELT occurred at ESO on December 3, 2014 (de Zeeuw, Tamai, \& Liske 2014). On May 25, 2016 ESO signed the contract for ELT Dome and Telescope Structure (ESO1617 - Organisation Release). An expanded view of the Universe (Science with the European Extremely Large Telescope) can be found in http://www.eso.org/sci/ facilities/eelt/docs/.

c) The James Webb Space Telescope (JWST) will be a giant leap forward in our quest to understand the Universe and our origins. JWST will examine every phase of cosmic history: from the first luminous glows after the Big Bang to the formation of galaxies, stars, and planets to the evolution of our own solar system (https://jwst.nasa.gov/ science.html).

d) The THESEUS mission is designed to vastly increase the discovery space of the high energy transient phenomena over the entirety of cosmic history (http://www.isdc.unige.ch/theseus/). The main scientific goals of the proposed mission are to:

i) Explore the Early Universe (cosmic dawn and reionization era) by unveiling a complete census of the Gamma-Ray Burst (GRB) population in the first billion years.

ii) Perform an unprecedented deep monitoring of the X-ray transient Universe. Figure 4 shows the GRASP FoV versus Effective Area) as function of energy (upper panel) and the cumulative distribution of GRBs with redshift determination as a function of the redshift for Swift (in $10 \mathrm{yr}$ ) and the prediction for THESEUS (in $3 \mathrm{yr}$ ) (lower panel).

e) e-ASTROGAM (enhanced ASTROGAM) is a breakthrough observatory space mission, with a detector composed by a silicon tracker, a calorimeter, and an anticoincidence system, dedicated to the study of the non-thermal Universe in the photon energy range from $0.3 \mathrm{MeV}$ to $3 \mathrm{GeV}$ - the lower energy limit can be pushed to energies as low as $150 \mathrm{keV}$, albeit with rapidly degrading angular resolution, for the tracker, and to $30 \mathrm{keV}$ for calorimetric detection. The mission is based on an advanced space-proven detector technology, with unprecedented sensitivity, angular and energy resolution, combined with polari-
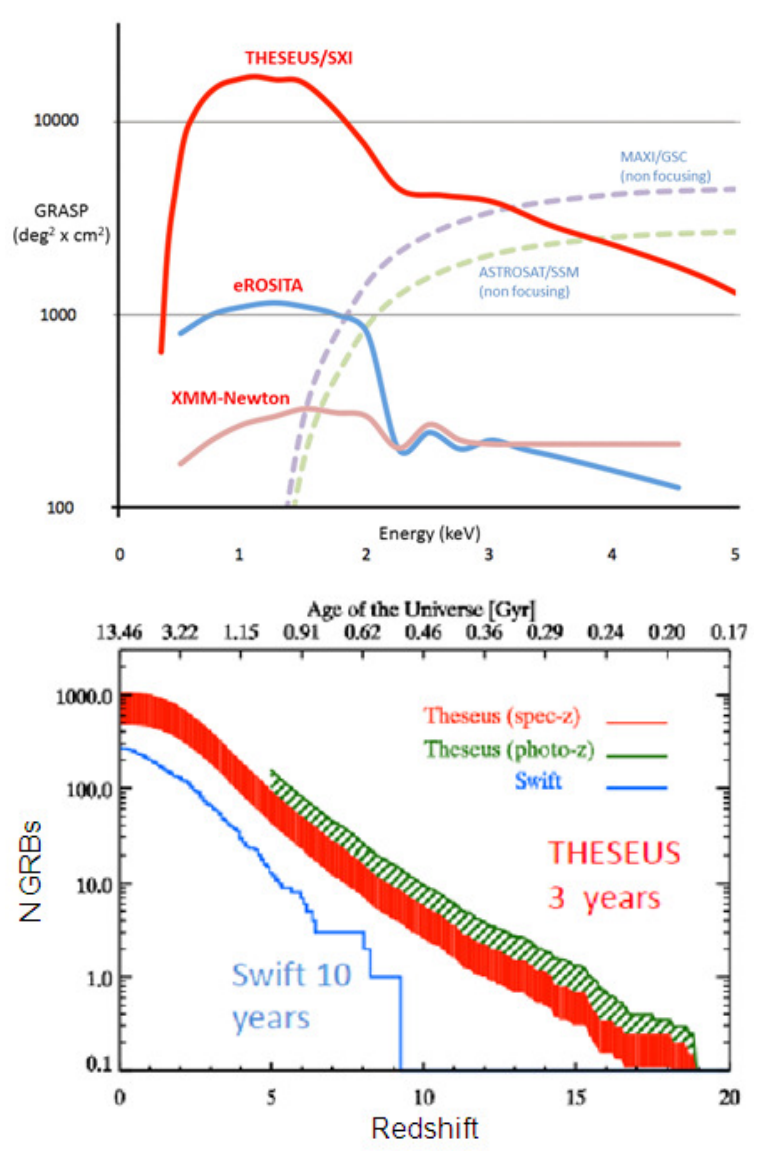

Fig. 4. (Upper panel: the GRASP (FoV versus Effective Area) as function of energy; lower panel: the cumulative distribution of GRBs with redshift determination as a function of the redshift for Swift (in 10 yr) and the prediction for THESEUS (in 3 yr) (http: //www.isdc.unige.ch/theseus/).

metric capability. Thanks to its performance in the $\mathrm{MeV}-\mathrm{GeV}$ domain, substantially improving its predecessors, e-ASTROGAM will open a new window on the non-thermal Universe, making pioneering observations of the most powerful Galactic and extragalactic sources, elucidating the nature of their relativistic outflows and their effects on the surroundings. With a line sensitivity in the $\mathrm{MeV}$ energy range one to two orders of magnitude better than previous generation instruments, e-ASTROGAM will determine the origin of key isotopes fundamental for the understanding of supernova explosion and the chemical evolution of our Galaxy. The mission will provide unique data of significant interest to a broad astronomical community, complementary to powerful observatories such as LIGO-Virgo-GEO600-KAGRA, SKA, ALMA, E-ELT, TMT, LSST, JWST, Athena, 

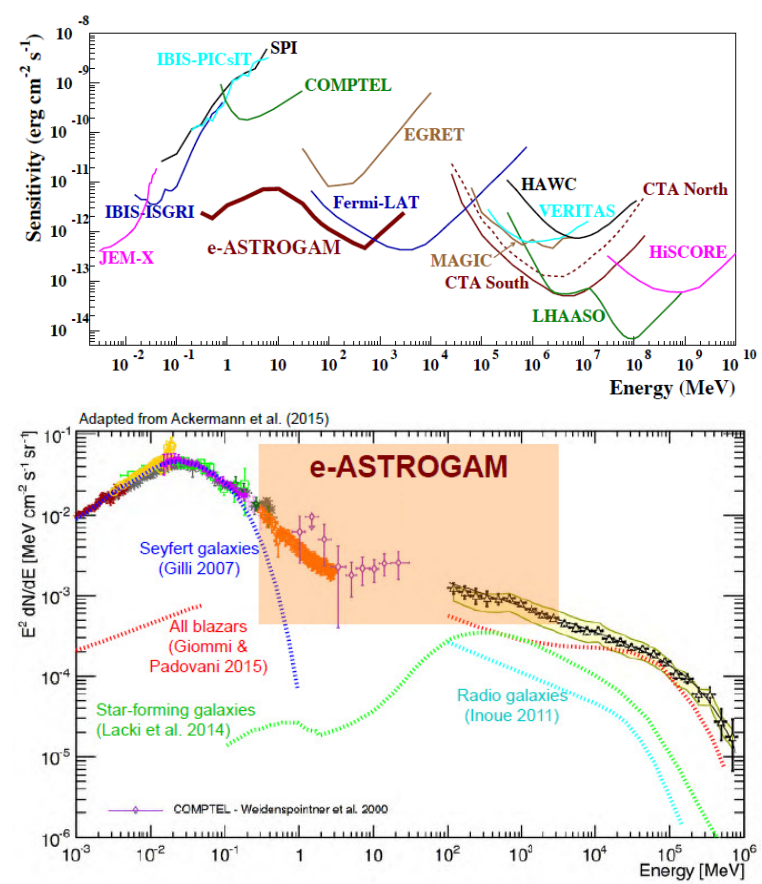

Fig. 5. Upper panel: the sensitivity of e-ASTROGAM compared with those of the past, present and future experiments; lower panel: the compilation of measurements of extragalactic sources between $1 \mathrm{keV}$ and $820 \mathrm{GeV}$, The semitransparent band indicates the energy region in which e-ASTROGAM will strongly improve on present knowledge (De Angelis et al. 2017).

CTA, IceCube, KM3NeT, and the promise of eLISA (De Angelis et al. 2017). Figure 5 shows: in the upper panel the sensitivity of e-ASTROGAM compared with those of the past, present and future experiments; in the lower panel the compilation of measurements of extragalactic sources between 1 $\mathrm{keV}$ and $820 \mathrm{GeV}$, The semitransparent band indicates the energy region in which e-ASTROGAM will strongly improve on present knowledge (De Angelis et al. 2017).

f) The GAMMA-400 gamma-ray telescope: the next absolutely necessary step in the development of extraterrestrial high-energy $\gamma$-ray astronomy is the improvement of the physical and technical characteristics of $\gamma$-ray telescopes, especially the angular and energy resolutions. Such a new generation telescope will be GAMMA-400, which will be installed onboard the Russian space observatory.

The GAMMA-400 gamma-ray telescope is intended to measure the fluxes of gamma-rays and cosmic-ray electrons and positrons in the energy range from $100 \mathrm{MeV}$ to several TeV. Such measurements concern the following scientific tasks: investi-

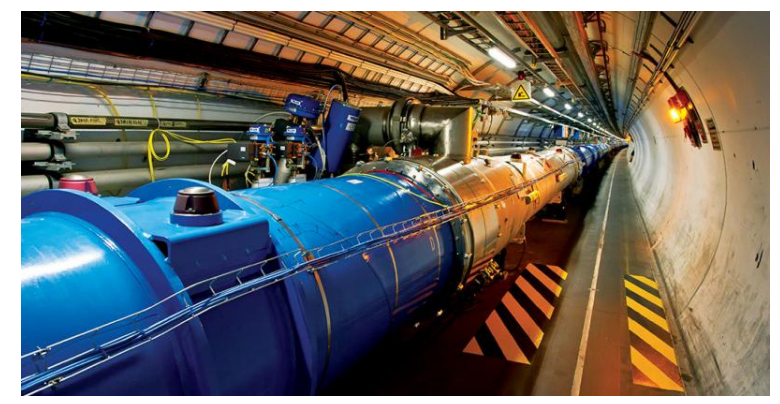

Fig. 6. The Large Hadron Collider is the world's largest and most powerful particle accelerator (Image: CERN, at https://home.cern/topics/ large-hadron-collider).

gation of point sources of gamma-rays, studies of the energy spectra of Galactic and extragalactic diffuse emission, studies of gamma-ray bursts and gammaray emission from the Sun, as well as high precision measurements of spectra of high-energy electrons and positrons. Also GAMMA- 400 instrument provides the possibility for protons and nuclei measurements up to knee. But the main goal for the GAMMA-400 mission is to perform a sensitive search for signatures of dark matter particles in high-energy gamma-ray emission (Topchiev et al. 2016a,b, and the references therein). GAMMA-400 will operate in the highly elliptic orbit continuously for a long time with the unprecedented angular $\left(\sim 0.01^{\circ}\right.$ at $\left.\mathrm{E}_{\gamma}=100 \mathrm{GeV}\right)$ and energy $\left(\sim 1 \%\right.$ at $\mathrm{E}_{\gamma}=100$ $\mathrm{GeV}$ ) resolutions better than the Fermi-LAT, as well as ground $\gamma$-ray telescopes, by a factor of 5-10. GAMMA-400 will permit to resolve $\gamma$-rays from annihilation or decay of dark matter particles, identify many discrete sources (many of which are variable), to clarify the structure of extended sources, and to specify the data on the diffuse emission (Topchiev et al. 2017).

g) The Large Hadron Collider (LHC) is the world's largest and most powerful particle accelerator (https://home.cern/topics/ large-hadron-collider). It first started up on 10 September 2008, and remains the latest addition to CERN's accelerator complex. The LHC consists of a $27 \mathrm{~km}$ ring of superconducting magnets with a number of accelerating structures to boost the energy of the particles along the way. Figure 6 shows a partial view of the tunnel hosting the accelerator.

The LHC will answer some of the fundamental open questions in physics, concerning the basic laws governing the interactions and forces among the elementary objects, the deep structure of space and 
time, and in particular the interrelation between quantum mechanics and general relativity. Data are also needed from high-energy particle experiments to suggest which versions of current scientific models are more likely to be correct-in particular to choose between the Standard Model and Higgs-less model and to validate their predictions and allow further theoretical development. Many theorists expect new physics beyond the Standard Model to emerge at the TeV energy level, as the Standard Model appears to be unsatisfactory. Issues explored by LHC collisions include i) the mass of elementary particles being generated by the Higgs mechanism; ii) supersymmetry, an extension of the Standard Model and Poincaré symmetry; iii) extra dimensions, as predicted by various models based on string theory; iv) the nature of the dark matter that appears to account for $\sim 27 \%$ of the mass-energy of the universe; v) answer to the question if the electroweak force and the strong nuclear force are just different manifestations of one universal unified force, as predicted by various Grand Unification Theories; vi) why the fourth fundamental force (gravity) is so many orders of magnitude weaker than the other three fundamental forces; vii) are there additional sources of quark flavour mixing, beyond those already present within the Standard Model?; viii) why are there apparent violations of the symmetry between matter and antimatter?; ix) what are the nature and properties of quark-gluon plasma, thought to have existed in the early universe and in certain compact and strange astronomical objects today? Some important results obtained with the LHC will be discussed later.

h) The BICEP (Background Imaging of Cosmic Extragalactic Polarization) and the Keck Array are a series of cosmic microwave background (CMB) experiments. They aim to measure the polarization of the $\mathrm{CMB}$; in particular, measuring the B-mode of the CMB. The experiments have had four generations of instrumentation, consisting of BICEP1, BICEP2, the Keck Array, and BICEP3. These experiments are observing from the South Pole, and their aims are to discover signatures of Inflation by actually detecting the Cosmic Gravitational Background (CGB) via its weak imprint as the unique B-mode polarization signature of the $\mathrm{CMB}$, directly probing the Universe at an earlier time than ever before. Each generation represents a large increase in sensitivity to B-mode polarization. BICEP1 observed from 2006-2008 with 98 detectors, BICEP2 began observing in the beginning of 2010 with 512 detectors, and the first three of five Keck Array telescopes began observing in the beginning of
2011, each with 512 detectors. The final two Keck Array receivers were deployed during the summer season of 2012. BICEP3, with a total of 2,560 detectors, has been operational since May 2016. The main goal of BICEP experiments is to test the validity of the theory of the Inflation (Keating et al. 2003; Ogburn IV et al. 2010). In particular, if Inflation happened immediately after the Big Bang, it would have produced turbulence in the structure of space-time itself-gravitational waves like the kind LIGO detected recently. While these waves would be too weak for LIGO to see, they would twist the orientation of the light, which is known as polarization. We will discuss later the results coming from BICEP2/Keck-Array and we will comment on their validity. Of course the list of big experiments is far to be complete, but it is enough to show to the reader the efforts that the international scientific community are facing both for determining the frontier scientific tests to validate the current theories and for the difficulties in providing sufficient budgets for their realization.

\subsection{Small experiments}

In this short excursion about the tools necessary for an advance of our knowledge of the physics of the Universe, we cannot omit the extreme importance of small experiments, like those Space-based: small-, mini-, micro-, nano-, and cube-satellites, and those Ground-based: small-telescope, and Robotic-telescopes.

Castro-Tirado (2010a) in his review presented a historical introduction to the field of Robotic Astronomy, discussing the basic definitions, the differing telescope control operating systems, observatory managers, as well as a few current scientific applications in that time.

The number of automatic astronomical facilities worldwide continues to grow, and the level of robotisation, autonomy, and networking is increasing as well. This has a strong impact in many astrophysical fields, like the search for extrasolar planets, the monitoring of variable stars in our Galaxy, the study of active galactic nuclei, the detection and monitoring of supernovae, and the immediate followup of highenergy transients such as gamma-ray bursts (CastroTirado 2008, 2010b).

The number of Robotic Autonomous Observatories (RAOs) has rapidly grown. Figure 7 shows the location of more than 100 RAOs worldwide (Castro Cerón 2011). They are providing excellent results which should be impossible to obtain with the larger telescopes subject to strict scheduling, and in any case not available for long term runs of observations. 


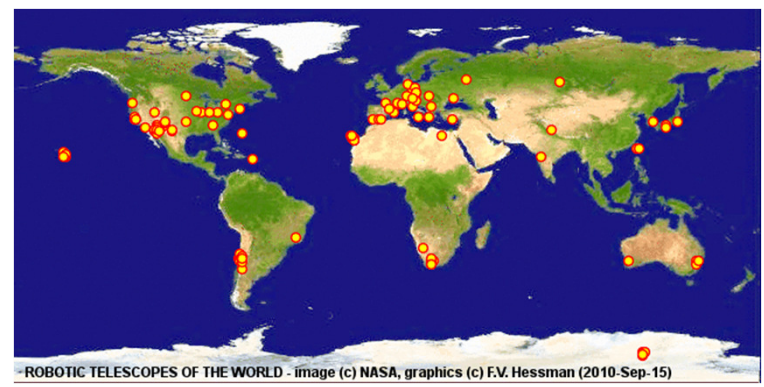

Fig. 7. The Robotic Autonomous Observatories worldwide (adopted from Castro Cerón (2011) after Hessman (2001a,b).

The most important news about the many scientific results obtained with the RAOs can be found in the proceedings of the series of Workshops on Robotic Autonomous Observatories (Bloom et al. 2010; Guziy et al. 2012; Tello et al. 2014; CaballeroGarcía et al. 2016).

Just for giving the reader a short panorama about the many small ground- and space-based experiments, not necessarily autonomous, we list the following:

a) MITSuME (Multicolor Imaging Telescope for Survey and Monstrous Explosions) has been built to perform Multi-color photometry of NIR/optical afterglow covering the wavebands from $\mathrm{K}_{s}$ to g' allowing the photometric redshift measurements up to $\mathrm{z} \sim 10$. Two $50 \mathrm{~cm}$ optical telescopes are built at Akeno, Yamanashi in eastern Japan, and at OAO, Okayama in western Japan. Each telescope has a Tricolor Camera, which allows us to take simultaneous images in g', $\mathrm{R}_{c}$, and $\mathrm{I}_{c}$ bands. These telescopes respond automatically to GCN alerts and start taking series of tricolor images, which are immediately processed through the analysis pipeline on site. The pipeline consists of source finding, catalog matching, sky coordinates mapping to the image pixels, and photometry of the found sources. An automated search for an optical counterpart is performed. While waiting for GRBs, the MITSuME Telescopes automatically patrol pre-selected interesting objects such as AGNs and galactic transients for multiwavelength studies with Fermi (GLAST) and MAXI (Shimokawabe et al. 2009).

b) The CHASE (CHilean Automatic Supernova sEarch) project began in 2007 (Pignata et al. 2009) with the goal to discover young, nearby southern supernovae in order to i) better understand the physics of exploding stars and their progenitors, and ii) refine the methods to derive extra-galactic distances. During the first four years of operation,
CHASE has produced more than 130 supernovae, being the most successful project of its type in the southern hemisphere (Hamuy et al. 2012).

c) The Russian global network of telescopes robot MASTER (Lipunov et al. 2010). MASTER is very fast positioning alert, follow up and survey twin telecopes Global network with own realtime auto-detection software. MASTER goal is One Sky in One Night up to 20-21 mag. The network is spread along the whole world. In the following are reported the MASTER Net Sites: i) MASTER-Amur: Russia, near Blagoveschensk. Blagoveschensk State Pedagogic University. ii) MASTER-Tunka: Russia, near Irkutsk. Applied Physics Institute, Irkutsk State University. iii) MASTER-Ural: Russia, near Ekaterinburg, Since 2008. Kourovka Astronomical Observatory, Ural State University. iv) MASTERKislovodsk: Russia, Near Kislovodsk. Kislovodsk Solar Station of the Pulkovo Observatory, Sternberg Astronomical Institute, Lomonosov Moscow State University. v) MASTER-SAAO: South Africa, Sutherland, since 2014. South African Astonomical Observatory (SAAO). vi) MASTER-IAC: Spain, Canarias Islands, since 2015 The Instituto de Astrofísica de Canarias (IAC). vii) MASTER-OAFA: Argentina, since 2012 Observatorio Astronomico Felix Aguilar (OAFA), Instituto de Ciencias Astronomicas de la Tierra y del Espacio (ICATE), National University of San Juan. viii) MASTERProgenitor: Russia, Moscow, Alexander Krylov Observatory, Since 2002.

d) Very small satellites for multifreqyency astrophysics have been discussed by Hudec et al. (2017). About the small satellites we can assist to a strong competition (typically for ESA missions, 60 proposals for 1 satellite), and moreover all the system is affected by funding problems. The development of the Pico (Cube) and Nanosatellites is running at many universities, mostly with involvement of students for evident goals of education. The standard size for a CubeSat is 1 liter volume, i.e. $10 \times 10 \times 10 \mathrm{~cm}^{3}$ and typically a weight of $\sim 1.3 \mathrm{~kg}$. Multiple modules are possible, i.e. 3 Units $=3$ modules/units, i.e. $10 \times 10 \times 30 \mathrm{~cm}^{3}$, typically up to 12 Units. The range of weight of Picosatellites is 0.1-1 kg, Femtosatellites 10-100 g, Nanosatellites 1$10 \mathrm{~kg}$, Microsatellites 10-100 kg. Recent technological progress allows their use in any field of astrophysics. Undoubtedly MASTER contributions to transient alerts in Astronomer's telegrams is fundamental. For instance in the period 2013-2014, MASTER contribution is of order $25 \%$ of the total as shown in Figure 8 (after Buckley 2015). Though we 


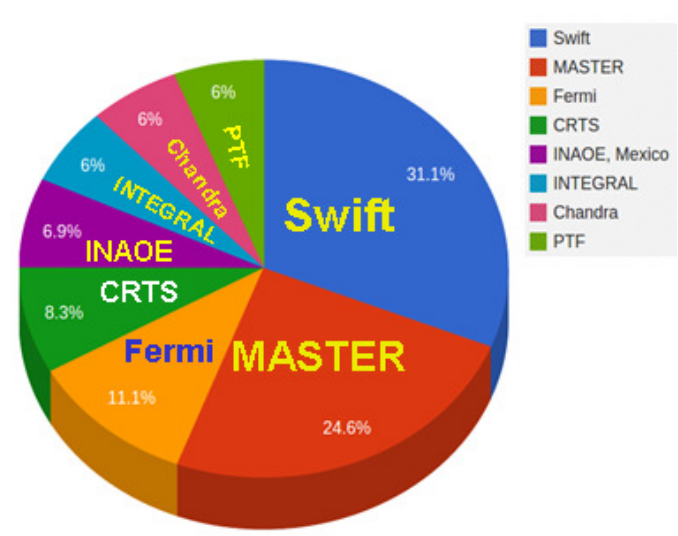

Fig. 8. Contribution of different space- and ground-based experiments to the transient alerts in Astronomer's telegrams in the period 2013-2014 (after Buckley 2015).

have not shown a complete list of small experiments both space- and ground-based, we are able to affirm that small telescopes are unreplaceable tools complementary to larger telescopes and to bigger groundand space-based multifrequency experiments.

\section{THE USE OF WISDOM IN PHYSICS}

Thanks to the numerous experiments groundand space-based, we have collected a huge amount of experimental data, the use of which is extremely difficult. This greatly limits the possibility of reaching a synthesis. In contrast, this immense amount of data generates a production of thousands of scientific articles that only in a few cases lead to a real advancement of knowledge.

The science policy, that is now dominating the scientific world, measures the value of a researcher according to: i) the number of "scientific" publications regardless of the real contribution they make to a substantial advancement of knowledge, and ii) the number of citations.

This system foments only the increase of the production of articles at the expense of the synthesis that would be needed, and a chain of "friendly" citations.

And this produces an absurd. Indeed, in order to write a scientific paper a "normal" scientist needs roughly three months of full time work. Thus, four papers in international refereed journals is the upper limit to the yearly publications. We can be generous adding half a dozen of papers presented at the international conferences. Then the new upper limit of publications can be of order of $10 \pm 3 \mathrm{yr}^{-1}$.

Therefore we can derive a severe question to all colleagues who produce more than 13 articles/year.

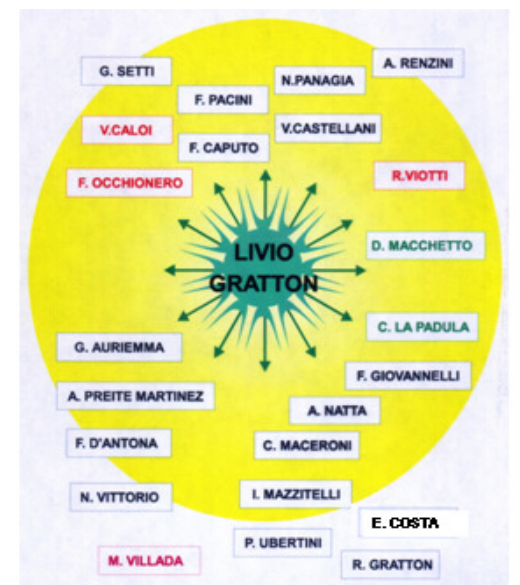

Fig. 9. The supernova "Livio Gratton" produced remnant-pupils, all of them rather well known within the international astrophysical community. (Giovannelli 2010).

How they do? There are many colleagues who publish more than 100 articles per year!

One of us (FG) remembers a repetitive suggestion of Livio Gratton - who was his professor of astrophysics at La Sapienza University of Roma-who felt a true incentive to scientific research: Favour the quality against the quantity! And he was completely right. Indeed he generated-like a supernova expelling heavy elements in the interstellar mediuma number of very famous pupils that pervaded the world of astrophysics. All the readers surely know at least two of them, sketched in Figure 9 (Giovannelli 2010).

After $\sim 44$ years experience about Multifrequency Astrophysics, we can affirm that: there are many problems in performing Simultaneous Multifrequency, Multisite, Multi-instrument, Multiplatform Measurements due to i) objective technological difficulties; ii) sharing common scientific objectives; iii) problems of scheduling and budgets; iv) politic management of science. In our opinion the most critical point is the latter which is moving on a "slippery ground". An example of this point is clearly illustrated by the SIXE: Spanish Italian Xray Experiment (Giovannelli et al. 1993). SIXE was planned as a multifrequency (X-ray, Optical) payload for Long-Term continuous observations of few selected cosmic sources in order to clearly understand the physics governing their behaviour. Later the phase-A of SIXE was completed thanks to a funding of the Spanish PNIE (Plan Nacional Investigación Espacial), being Principal Investigators Jordi Isern and Franco Giovannelli, and Lola Sabau- 


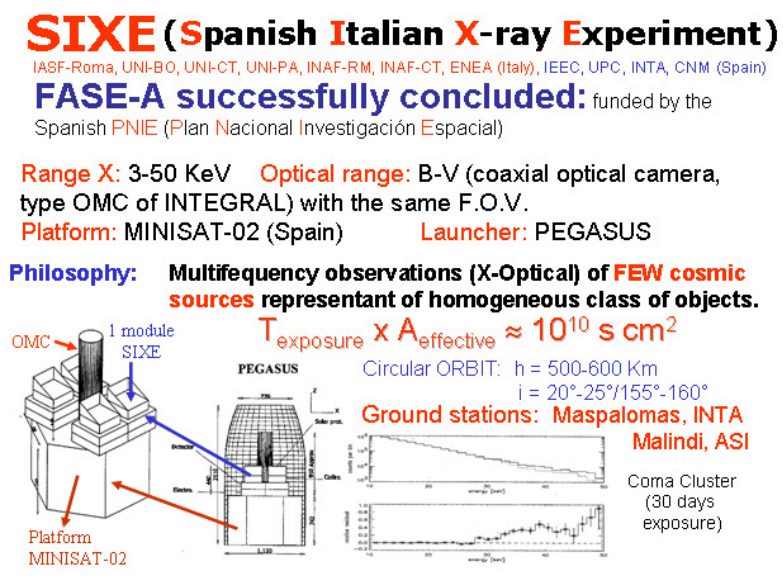

Fig. 10. SIXE (Spanish Italian X-ray Experiment): main characteristics.

Graziati as first Co-I (PNIE-CICYT Report, ESP971784-E grant: Isern et al. 1999a; Giovannelli et al. 2002a).

Summaries of that report can be found in several papers later published (Isern et al. 1999b; Giovannelli et al. 1999a,b, 2001, 2002b). Figure 10 shows a summary of the main characteristics of SIXE.

SIXE was submitted to ASI (Agenzia Spaziale Italiana: Italian Space Agency) in order to ask a funding for sharing the costs of the payload with the PNIE, being the launcher (PEGASUS) provided by Spain. No answer at all!!!

After about 25 years from the original idea (Giovannelli, F., Sabau-Graziati, L. et al. 1993) SIXE papers are still read: up till now more than 1500 readings from all the World (source: Research Gate). It is the most read paper in all INAF Institutes!!!

And now some examples of problems resolved with the help of multifrequency observations and good small quantities of wisdom in physics.

\subsection{X-ray/Be systems}

$\mathrm{X}$-ray/Be systems are formed by a compact star and an optical star. Obviously there is a mutual influence between the two stars. Low-energy (LE) processes influence high-energy (HE) processes and vice versa. Never confuse the effect with the cause. There is a general law in the Universe: Cause and Effect. The Cause generates an Effect and NOT vice versa!

Time-lag between HE events and LE events in disk-fed accreting X-ray binaries (XRBs) has been noted in many systems, but the trigger of the work resulted in a model for explaining in general such a phenomenon (Bisnovatyi-Kogan \& Giovannelli 2017) was given by Giovannelli \& Sabau-Graziati (2011) who noted a systematic delay between the relative enhancement in luminosity of the optical Be staroccurring at the periastron passage of the neutron star - and the subsequent X-ray flare in the system HDE 245770/A $0535+26$. The model for such a system was developed and corroborated by many events (Giovannelli, Bisnovatyi-Kogan, \& Klepnev 2013: GBK13), and later by events reported in Giovannelli et al. (2015) where also a relationship between $\Delta V_{\text {mag }}$ of the optical star at the periastron and X-ray intensity $\left(\mathrm{I}_{\mathrm{X}}\right)$ of the 8-day delayed flare was produced.

Briefly, the model based on an accretion disk geometrically thin and optically thick without advection (Shakura \& Sunyaev 1973; Bisnovatyi-Kogan 2002) is the following: in the vicinity of periastron the mass flux $\dot{M}$ increases (depending on the activity of the Be star) between $\approx 10^{-8}$ and $\approx 10^{-7} \mathrm{M}_{\odot} \mathrm{yr}^{-1}$. The outer part of the accretion disk becomes hotter, therefore the optical luminosity $\left(\mathrm{L}_{\mathrm{opt}}\right)$ increases. Due to large turbulent viscosity, the wave of the large mass flux is propagating toward the neutron star, thus the X-ray luminosity $\left(\mathrm{L}_{\mathrm{x}}\right)$ increases due to the appearance of a hot accretion disk region and due to the accretion flow channeled by the magnetic field lines onto magnetic poles of the neutron star. The time-delay $\tau$ is the time between the optical and Xray flashes appearance.

It is right to remind that the mechanism proposed by GBK13 for explaining the X-ray-optical delay in A $0535+26 / \mathrm{HDE} 245770$ is based on an enhanced mass flux propagation through the viscous accretion disk. This mechanism, known as UVoptical delay (the delay of the EUV flash with respect to the optical flash) was observed and modeled for cataclysmic variables (e.g. Smak 1984; Lasota 2001). Time delays have been detected also in several other X-ray transient binaries. This is the reason that urged Bisnovatyi-Kogan \& Giovannelli (2017) to generalize the aforementioned model, developed for the particular case of A $0535+26 / \mathrm{HDE}$ 245770 (Flavia' star). This general model provides the formula (1) of the time delay in transient cosmic accreting sources:

$$
\tau=6.9 \frac{\mathrm{m}^{2 / 3} \dot{\mathrm{m}}^{1 / 15}}{\alpha^{4 / 5}\left(\mathrm{~T}_{4}\right)^{28 / 15}}
$$

where:

$$
\begin{aligned}
& \mathrm{m}=\mathrm{M} / \mathrm{M}_{\odot} ; \quad \dot{\mathrm{m}}=\dot{\mathrm{M}} /\left(10^{-8} \mathrm{M}_{\odot} / \mathrm{yr}\right) ; \\
& \mathrm{T}_{4}=\mathrm{T}_{0} / 10^{4} \mathrm{~K} ; \quad \alpha=\text { viscosity }, \text { and } \\
& \mathrm{T}_{0}=\text { maximum temperature in optics. }
\end{aligned}
$$




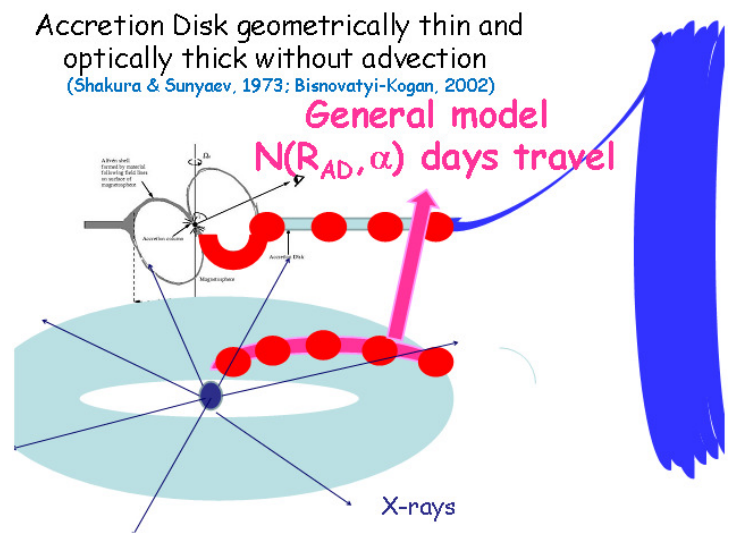

Fig. 11. Time-lag general model for disk-fed accreting XRBs (Bisnovatyi-Kogan \& Giovannelli 2017).

By using this formula it is possible to obtain an excellent agreement between the experimental and theoretical delays found in:

- X-ray/Be system A0535+26/HDE245770: $\tau_{\text {exp }} \simeq 8$ days $(\mathrm{GBK} 13) ; \tau_{t h} \simeq 8$ days;

- Cataclysmic variable SS Cygni; $\tau_{\text {exp }}=0.9-1.4$ days (Wheatley, Mauche \& Mattei 2003); $\tau_{t h} \simeq$ 1.35 days;

- Low-mass X-ray binary Aql X-1/V1333 Aql: $\tau_{\text {exp }} \sim 3$ days (Shahbaz et al. 1998); $\tau_{t h} \simeq$ 3.2 days

- Black hole X-ray transient GRO J1655-40: $\tau_{\text {exp }} \sim 6$ days (Orosz et al. 1997); $\tau_{t h} \simeq 6.5$ days.

In this general formula the $\alpha$-viscosity parameter plays an important role, and usually it is hard to be determined. However, if the other parameters are known, because experimentally determined, the formula (1) can be used for determining $\alpha$, taking into account the experimental delay measured in a certain source.

This general model for the time-lag for disk-fed accreting XRBs is sketched in Figure 11.

Another example of the use of wisdom is that referred to the X-ray/Be system A 1118-61/Hen 3640, for which Reig, Fabregat \& Coe (1997) used one single measurement of the equivalent width (EW) of $\mathrm{H}_{\alpha}(89 \AA)$ in their interesting diagram in which a relationship between the $\mathrm{H}_{\alpha}$-EWs and the orbital period $\left(\mathrm{P}_{\text {orb }}\right)$ of $\mathrm{Be} / \mathrm{X}$-ray binaries has been found. The point relative to A 1118-61/Hen 3-640 system (red cross in Figure 12) is clearly outside of the line best fitting the other data. However, if we use the

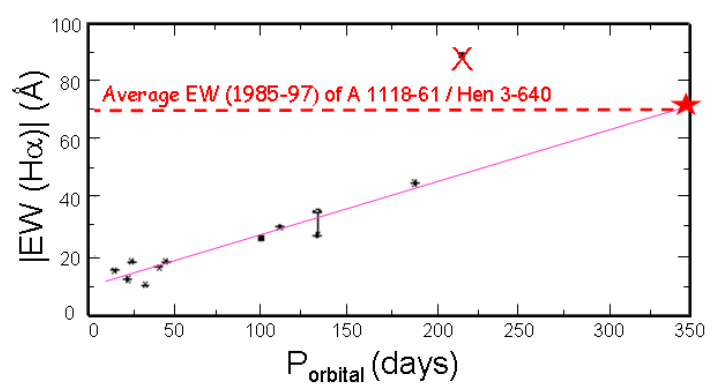

Fig. 12. The relationship between $\mathrm{H}_{\alpha}-\mathrm{EW}$ and $\mathrm{P}_{\text {orb }}$ in X-ray/Be systems (after Reig, Fabregat, \& Coe 1997; Villada et al. 1999).

average value (from 1985 to 1997) of $\mathrm{H}_{\alpha}-\mathrm{EW}=70$ A, like reported in Villada et al. (1999), also the position of the A 1118-61/Hen 3-640 system is well on the line best fitting the data (red star in Figure 12). Moreover an indication of the possible value of the orbital period ( $\sim 350$ days), not yet known, is coming from that diagram. This can help the search for the orbital period of the system around the value of 350 days.

\subsection{The classical $T$ Tauri star RU Lupi}

A long-term (1982-1988) multifrequency program on Classical T Tauri Stars (CTTSs) was developed by the international group led by F. Giovannelli. The facilities used for such a campaign were the International Ultraviolet Explorer (IUE), the ASTRON Xray/UV Soviet satellite, and the ESO 0.6-m UBVRI telescope, 1-m IR telescope, 1.5-m telescope for low resolution optical spectroscopy, and 3.6-m telescope for Echelle high resolution spectroscopy.

The results were published in two main papers, the first with the experimental results (Giovannelli et al. 1995), the second with the interpretation of data and modeling (Lamzin et al. 1996). A review paper about RU Lupi was published by Giovannelli (1994).

One of the main results obtained during the longterm multifrequency program was the simultaneous detection of emissions in different energy bands that allowed to construct the Spectral Energy Distribution (SED) of RU Lupi, as shown in Figure 13.

In two occasions, RU Lupi showed a strong activity (Flare-Like Events: FLEs), much higher than that in "quiescence". Together with the FLEs reported in the literature, these two FLEs allowed to determine their periodicity: $\mathrm{P}_{\mathrm{FLEs}}=27.686 \pm 0.002$ days (Giovannelli 1994). This could be the rotational period of RU Lupi. Indeed, if we use the relationship between the X-ray luminosity $\left(\mathrm{L}_{\mathrm{X}}\right)$ versus the 


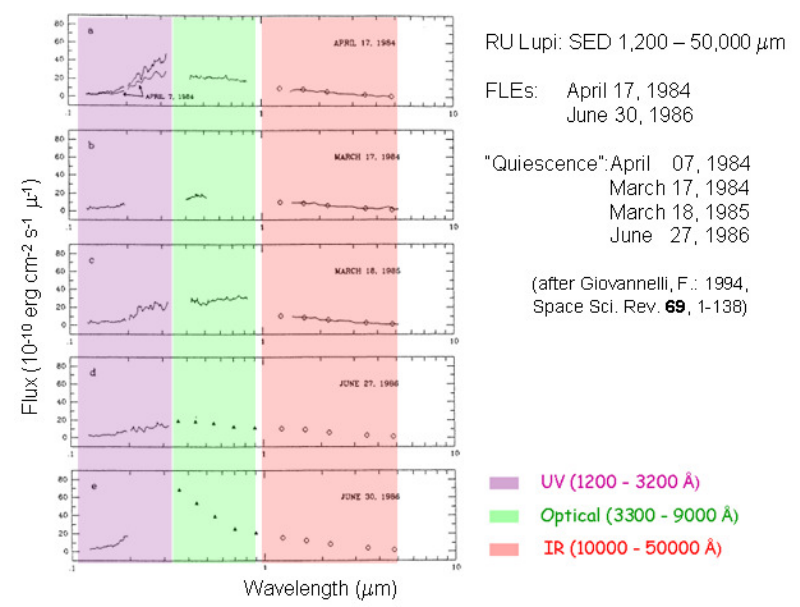

Fig. 13. SED $(1,200-50,000 \mu \mathrm{m})$ of RU Lupi in different epochs (after Giovannelli 1994).

rotational velocity $\left(\mathrm{v}_{\mathrm{rot}}\right)$ for $\mathrm{T}$ Tauri stars, late-type dwarfs, dKe-dMe stars, and RS CVn systems (Bouvier 1990), the position of RU Lupi fits the relation$\operatorname{ship} \log L_{\mathrm{X}}=27.2+2 \log v_{\text {rot }}$ if $\mathrm{P}_{\mathrm{FLEs}}=27.686 \pm$ 0.002 days is used, instead of using the "wrong" value reported in the literature of 3.7 days - that simply does not exist (Giovannelli et al. 1991) whose wrong origin is largely commented in the paper by Giovannelli (1994). Figure 14 shows the diagram of the X-ray luminosity $\left(\mathrm{L}_{\mathrm{X}}\right)$ versus the rotational velocity $\left(\mathrm{v}_{\mathrm{rot}}\right)$ where the "correct" position of RU Lupi is overlapped with a red cross, and the "wrong" position with the blue cross.

\subsection{Great example of synergy between Astrophysics and History}

Aschenbach modified Sedov's relation for determining the age of a SNR (Aschenbach 2016). He used as test the SNR Vela Jr (RX 0852.0-3946) discovered during the ROSAT All-Sky-Survey in Xrays (Aschenbach 1998) - and he gave an age of $\mathrm{T}_{\text {Aschenbach }} \sim 725 \mathrm{yr}$ - contrary to $\mathrm{T}_{\text {Sedov }} \sim 1714 \mathrm{yr}$, and a distance of $\sim 386 \mathrm{pc}$.

Historical document (Tatsunokuchi Persecution of Nichiren Daishonin "the Buddha of the last day of the law") supports this result with an exceptional precision: The date of the explosion was 12 September $1271(1 \pm 2 \mathrm{am}$-between the hours of the rat and the ox) (Soka Gakkai, The writings of Nichiren Daishonin Vol. I, p. 196). How is it possible to affirm that the explosion of the SN Vela Jr happened in that date with a strong precision?

The answer, indeed, can be found in the writings of Nichiren Daishonin. This buddhist monk presented to the public authority the "Risho Ankoku

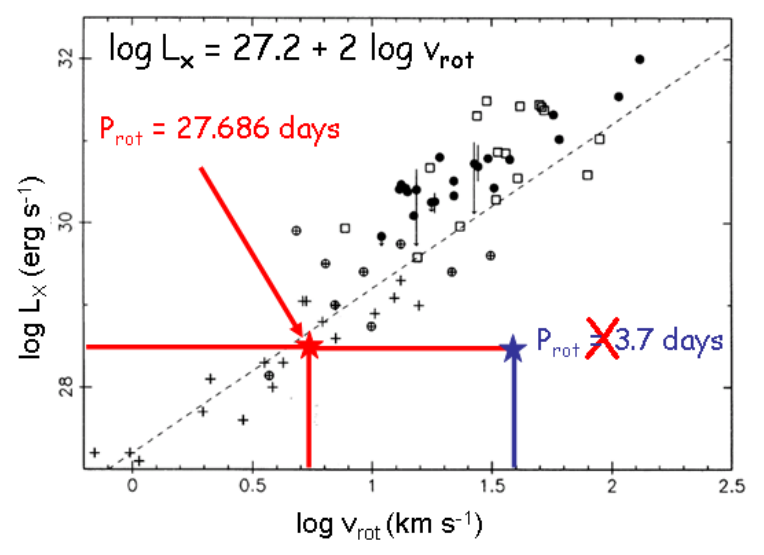

Fig. 14. X-ray luminosity vs stellar equatorial velocity for TTS $(\bullet)$, late-type dwarfs $(+)$, dKe-dMe stars $(\oplus)$, and RS CVn systems (square). The right position of RU Lupi is marked with a red cross, and the wrong position is marked with a blue cross (Giovannelli 1994 after Bouvier 1990).

Ron" (Establishing the Correct Teaching for the peace in the country) three times: a strong and clear critic to the behaviour of authority. For this reason he was persecuted and sentenced to death.

At that moment Nichiren was about to be beheaded, a luminous object (full Moon) shot across the sky, brightly illuminating the surroundings. The executioner fell on his face, his eyes blinded. The soldiers were filled with panic. Terrified, the soldiers called off the execution. This happened on the twelfth day of the ninth month of 1271 , between the hours of the rat and the ox (11:00 pm to 3:00 am). The event culminated $10^{\circ}$ above the horizon, celestial declination $-46^{\circ}$ (position of Vela $\mathrm{Jr}$ ).

\section{THE PRESENT SITUATION ABOUT THE KNOWLEDGE OF THE PHYSICS OF OUR UNIVERSE}

Undoubtedly the advent of new generation experiments ground- and space-based have given a strong impulse for verifying current theories, and for providing new experimental inputs for developing a new physics for going, probably, over the standard model (SM). Recent results coming from Active Physics Experiments (APEs) and Passive Physics Experiments (PPEs) have opened such a new path.

An extensive review on the situation about the knowledge of the physics of our Universe has been recently published by Giovannelli \& Sabau-Graziati (2016a). The reader interested is invited to look at that paper. However, we are obliged to discuss a 
few topics that, in our opinion, could be useful for a better understanding of the open problems still existing in the modern astrophysics.

\subsection{Active physics experiments}

One of the most exciting results from LHC is the detection of the Higgs boson which is often called "the God particle" because it's said to be what caused the "Big Bang" that created our Universe. Matter obtains mass interacting with Higgs field. Thus, if the Higgs Boson is detected, the Standard Model of Physics would be completed.

The Standard Model of particle physics takes quarks and leptons to be fundamental elementary particles, and describes the forces that govern their interactions as mediated through the exchange of further elementary particles. The exchanged particles are photons in the case of the electromagnetic interaction, $\mathrm{W}$ and $\mathrm{Z}$ bosons in the case of the weak interaction, and gluons in the case of the strong interaction. After the discovery of the W and $\mathrm{Z}$ bosons in the early 1980s, the elucidation of the mechanism by which they acquire mass became an important goal for particle physics. Within the Standard Model the $\mathrm{W}$ and $\mathrm{Z}$ bosons have masses generated via the symmetry breaking Englert-BroutHiggs-Guralnik-Hagen-Kibble mechanism, proposed in 1964 and giving rise to a massive scalar particle, the Standard Model Higgs boson (Jakobs \& Seez 2015).

The hunt to Higgs boson started a few years ago with the most powerful accelerators constructed in the world, in particular with the different experiments of the LHC. These experiments can provide information about the first moment of the life of the Universe. LHC is a complementary tool for HE observatories looking directly to the Universe.

The Higgs boson discovery was announced by the ATLAS (A Toroidal LHC ApparatuS) and CMS (Compact Muon Solenoid) collaborations on 4th July 2012. Evidence for a new particle with the mass of about $125 \mathrm{GeV}$ and the properties of the Standard Model Higgs boson.

From ATLAS results, a $5.0 \sigma$ excess at $\sim 126.5$ $\mathrm{GeV}$ has been detected. This value is compatible with the expected mass of Higg's boson (Gianotti 2012; Aad et al. 2012). The Compact Muon Solenoid (CMS) experiment at LHC detected a new boson at $125.3 \pm 0.6 \mathrm{GeV}$ with $4.9 \sigma$ significance (Incandela 2012; The CMS Collaboration 2012a). This result, together with that from ATLAS, if confirmed, would complete the SM of physics.

Thanks to collisions at $13 \mathrm{TeV}$ the experiment Large Hadron Collider beauty (LHCb) at LHC de- tected a new particle: the Pentaquark. The existence of the pentaquark was theoretically suggested since 1960-ies (Gell-Mann 1964). Pentaquark gives a new way for the combination of the quarks that are the fundamental constituents of neutrons and protons (Cardini 2015; Aaij et al. 2015).

\subsection{Passive physics experiments}

One of the most important questions still open is the search for experimental proof of the inflation. The expansion is thought to have been triggered by the phase transition that marked the end of the preceding grand unification epoch at $\approx 10^{-36} \mathrm{~s}$ after the Big Bang. It is not known exactly when the inflationary epoch ended, but it is thought to have been between $\approx 10^{-33} \mathrm{~s}$ and $\approx 10^{-32} \mathrm{~s}$ after the Big Bang. The experimental proof of the inflation could come from measurements of Cosmic Microwave Background (CMB) polarization. Winstein (2007, 2009) discussed the problem of CMB polarization in the following decade.

We know from the theory that linear polarization of the CMB photons is induced via Thomson scattering by quadrupole anisotropy at recombination that occurred at $\mathrm{z} \sim 1100$ corresponding to $\mathrm{t} \sim 1.2 \times 10^{13}$ $\mathrm{s}$ after the Big Bang. In turn, quadrupole anisotropy is induced by: i) density perturbations (scalar relics of inflation) producing a curl-free polarization vector field (E-modes); ii) gravitational waves (tensor relics of inflation) producing both curl-free and curlpolarization fields (B-modes).

No other sources for a curl-polarization field on the CMB at large angular scales exist. Thus, Bmodes are a clear signature of inflation (e.g. de Bernardis 2014).

Recently the collaboration of the BICEP2 experiment claims the detection of E-mode (Crites et al. 2015) and B-mode polarization of the CMB at at 7.0 $\sigma$ significance (Ade et al. 2015). If B-mode polarization would be confirmed, the inflationary model of the Universe would be definitively confirmed. However, big discoveries need big confirmations. For a robust detection of $\mathrm{B}$-modes, independent measurements and precise measurements of polarized foregrounds are mandatory.

Indeed, a key element to the primordial interpretation advanced by the BICEP2 team was excluding an explanation based on polarized thermal dust emission from our galaxy (Bucher 2015). An independent analysis cast doubt on the BICEP2 claim (Flauger, Hill, \& Spergel 2014). In September 2014 the Planck team published a paper on the level of polarized dust emission measured across the whole 
sky, and in particular in the BICEP2 field (Planck Collaboration 2014). This work also extrapolated the polarized dust signal seen in the Planck $353 \mathrm{GHz}$ map (a frequency in the Wien tail of the CMB blackbody where dust dominates) down to $150 \mathrm{GHz}$ and reached the conclusion that the BICEP B mode signal could be entirely explained by polarized dust emission although a primordial B mode contribution could not be ruled out.

However, the theory of inflation is criticized by Ijjas, Steinhadt \& Loeb (2013) after Planck 2013 results. They suggest that the origin of the Universe is not the Big Bang, but could be a "bouncing" Universe that does not need inflation. MembraneUniverses that clashed endlessly could be a "plausible" alternative model for the Universe (Erickson et al. 2007; Steinhardt, Turok, \& Starkman 2008). Cyclic models of the universe have the advantage of avoiding initial conditions problems related to postulating any sort of beginning in time (Ijjas 2016).

For all these reasons is even more important to find an experimental proof of the Inflation.

\subsection{Confirmation of the Theory of General Relativity}

In the last few years two further experimental results confirmed the validity of the theory of General Relativity (GR theory).

\subsubsection{Gravitational lenses}

Kochanek (2003) discussed "The whys and hows of finding 10,000 lenses", mentioning the first radio lens survey - the MIT Green Bank survey (MG)that found lenses by obtaining Very Large Array (VLA) snapshot images of flux-limited samples of $5 \mathrm{GHz}$ radio sources. The Hubble Space Telescope (HST), and Chandra observations (e.g. Dai \& Kochanek 2005) showed without any doubt that the gravitational lensing is operating.

Gravitational lensing is widely and successfully used to study a range of astronomical phenomena, from individual objects, like galaxies and clusters, to the mass distribution on various scales, to the overall geometry of the Universe (Williams \& Schechter 1997). They describe and assess the use of gravitational lensing as "gold standards" in addressing one of the fundamental problems in astronomy, the determination of the absolute distance scale to extragalactic objects, namely the Hubble constant.

Several papers have been published about the strong gravitational lensing (e.g. Tyson, Kochanski \& Dell'Antonio 1998; Tyson 2000 and references therein), and the weak gravitational lensing
(Wittman et al. 2000). A review on "Gravitational Lenses" have been published by Blandford \& Kochanek (2004). A book on "Gravitational Lensing: Strong, Weak and Micro" was published by Meylan et al. (2006). Winn, Rusin \& Kochanek (2004) reported the most secure identification of a central image, based on radio observations of PMN J1632-0033.

Therefore, a further dowel supports the GR theory.

\subsubsection{Gravitational waves}

The Universe that contains by definition all the matter or all the energy available showed one important event that was possible to be detected on the Earth. This event was a further direct experimental demonstration of the validity of the GR theory. Indeed, on September 14, 2015 at 09:50:45 UTC the two detectors of the Laser Interferometer Gravitational-Wave Observatory (LIGO) simultaneously observed a transient gravitational-wave signal. It matches the waveform predicted by GR theory for the inspiral and merger of a pair of black holes and the ringdown of the resulting single black hole. The signal was observed with a significance $\geq 5.1 \sigma$ (Figure 15). The source lies at a luminosity distance of $410_{-180}^{+160} \mathrm{Mpc}$ corresponding to a redshift $\mathrm{z}=0.090_{-0.04}^{+0.03}$. In the source frame, the initial black hole masses are $36_{-4}^{+5} \mathrm{M}_{\odot}$ and $29 \pm 4 \mathrm{M}_{\odot}$, and the final black hole mass is $62 \pm 4 \mathrm{M}_{\odot}$ with $3.0 \pm 0.5$ $\mathrm{M}_{\odot} \mathrm{c}^{2}$ radiated in gravitational waves. All uncertainties define $90 \%$ credible intervals. These observations demonstrate the existence of binary stellarmass black hole systems. This is the first direct detection of gravitational waves and the first observation of a binary black hole merger (Abbott et al. 2016a).

Abbott et al. (2016b) reported the second observation of a gravitational-wave signal produced by the coalescence of two stellar-mass black holes. The signal, GW151226, was observed by the twin detectors of the LIGO on December 26, 2015 at 03:38:53 UTC. The signal was detected at significance $\geq 5 \sigma$. The inferred source-frame initial black hole masses are $14.2_{-3.7}^{+8.3} \mathrm{M}_{\odot}$ and $7.5 \pm 2.3 \mathrm{M}_{\odot}$, and the final black hole mass is $20.8_{-1.7}^{+6.1} \mathrm{M}_{\odot}$. We find that at least one of the component black holes has spin greater than 0.2. This source is located at a luminosity distance of $440_{-190}^{+180} \mathrm{Mpc}$ corresponding to a redshift $\mathrm{z}=0.09_{-0.04}^{+0.03}$. All uncertainties define a $90 \%$ credible interval. This second gravitational-wave observation provides improved constraints on stellar populations and on deviations from the GR theory. 


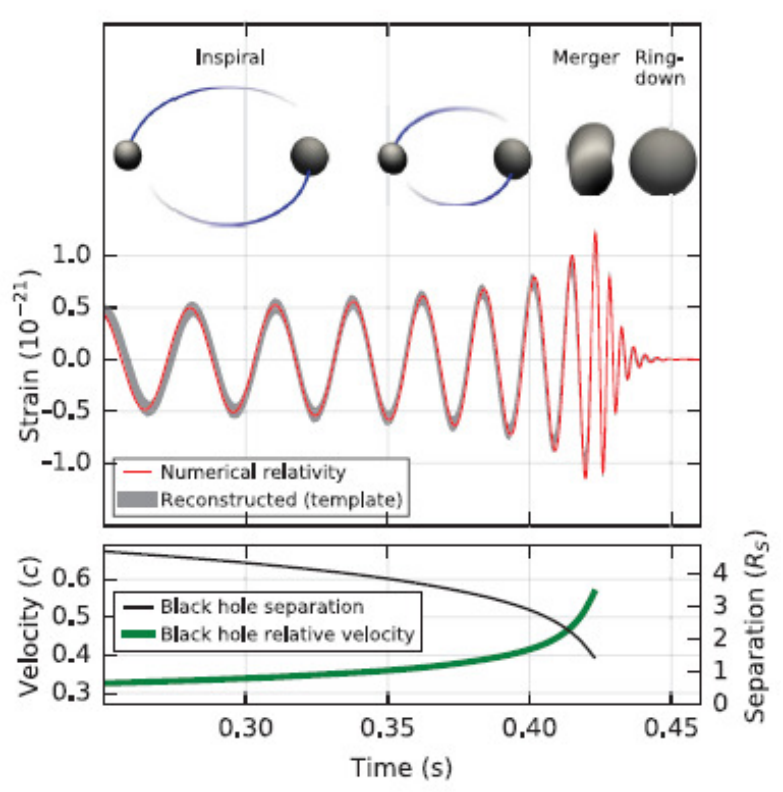

Fig. 15. The GW150914 event. Top: estimated gravitational-wave strain amplitude. Bottom: the Keplerian effective black hole separation in units of Schwarzschild radii (adopted from Abbott et al. 2016a).

For these detections of gravitational waves - first predicted by Einstein 100 years ago-Rainer Weiss, Barry Barish \& Kip Thorne have been awarded the 2017 Nobel prize in physics.

Abbott et al. (2016c) present a possible observing scenario for the Advanced LIGO (aLIGO) and Advanced Virgo gravitational-wave detectors over the next decade, with the intention of providing information to the astronomy community to facilitate planning for multi-messenger astronomy with gravitational waves.

Gravitational waves provide a revolutionary tool to investigate yet unobserved astrophysical objects. Especially the first stars, which are believed to be more massive than present-day stars, might be indirectly observable via the merger of their compact remnants. An interesting paper by Hartwig et al. (2016) developed a self-consistent, cosmologically representative, semi-analytical model to simulate the formation of the first stars. They estimated the contribution of primordial stars to the merger rate density and to the detection rate of the aLIGO. Owing to their higher masses, the remnants of primordial stars produce strong GW signals, even if their contribution in number is relatively small. They found a probability of $\geq 1 \%$ that the current detection GW150914 is of primordial origin. The higher masses of the first stars boost their GW signal, and therefore their detection rate. Up to five detections per year with aLIGO at final design sensitivity originate from Pop III BH-BH mergers. Approximately once per decade, we should detect a BH-BH merger that can unambiguously be identified as a Pop III remnant.

On 2017 August 17 the merger of two compact objects with masses consistent with two neutron stars was discovered through gravitational-wave (GW170817), gamma-ray (GRB 170817A), and optical (SSS17a/AT2017gfo) observations. The optical source was associated with the early-type galaxy NGC 4993 at a distance of just $\sim 40 \mathrm{Mpc}$, consistent with the gravitational-wave measurement, and the merger was localized to be at a projected distance of $\sim 2 \mathrm{kpc}$ away from the galaxy's center (Abbott et al. 2017a,b).

Lipunov et al. (1995) predicted the NS-NS merger at a distance of $\leq 50 \mathrm{Mpc}$ and the possibility of detecting GWs!

This prediction was born by the "Scenario Machine" that describes the evolution of gravimagnetic rotators (Lipunov 1987; Lipunov \& Postnov 1988), and recently commented by Giovannelli (2016).

On August 17, 2017 Multimessenger Astrophysics born! As pioneers of the Multifrequency Astrophysics, we are particularly happy!

\subsection{The accelerating Universe}

The discovery of the accelerating expansion of the Universe is a milestone for cosmology. A very interesting paper about this argument has been published in 2011 by the "Class for Physics of the Royal Swedish Academy of Sciences" as Scientific Background on the Nobel Prize in Physics 2011. In this paper a historical journey about the last century development of cosmology is brilliantly presented.

The discovery in 1998 that the universe is speeding up and not slowing down (Riess et al. 1998; Perlmutter et al. 1999) opened a question about the possibility of having different phases of acceleration and deceleration of the Universe along its life. Turner \& Riess (2002) from observations of SN 1997ff at z 1.7 favor the accelerating universe interpretation and provide some direct evidence that the universe was once decelerating. They show that the strength of this conclusion depends upon the nature of the dark energy causing the present acceleration. Only for a cosmological constant is the $\mathrm{SNe}$ evidence definitive. Using a new test which is independent of the contents of the universe, they show that the SN data favor recent acceleration $(z<0.5)$ and past deceleration $(\mathrm{z}>0.5)$.

Nielsen, Guffanti \& Sarkar (2016) found marginal evidence for cosmic acceleration from type Ia Super- 


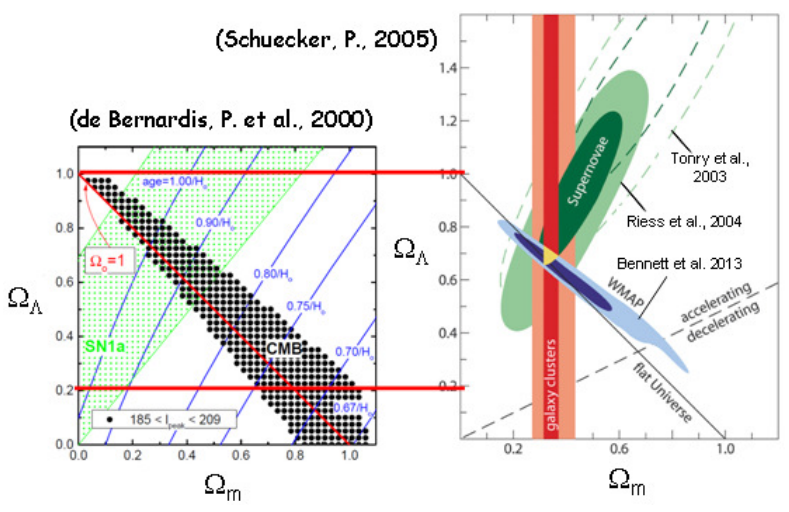

Fig. 16. Constraints of cosmological parameters (after de Bernardis et al. 2000; Schuecker 2005, Bennett et al. 2013).

novae. On the contrary, Haridasu et al. (2017) found that the SN data alone indicate an accelerating Universe at more than $4.56 \sigma$ confidence level.

\subsection{The Big Bang Nucleosynthesis theory has been proved}

The Big Bang Nucleosynthesis (BBN) theory predits the presence of a fixed content of light elements, the temperature of the Universe inversely proportional to the typical distance between galaxy clusters: $\mathrm{T}=\mathrm{T}(0)(1+\mathrm{z})$, and the $\mathrm{CMB}$ radiation temperature of $\sim 2.7 \mathrm{~K}$ (for details see Giovannelli \& Sabau-Graziati 2016a).

\subsection{Is the Universe Flat?}

One of the most critical points about our Universe is the problem of its flatness. The present state of the cosmological tests is illustrated in Figure 16.

The left panel of Figure 16 shows the results obtained with the BOOMERanG (Balloon Observations Of Millimetric Extragalactic Radiation and Geomagnetics) experiment (de Bernardis et al. 2000). They are fully consistent with a spatially flat Universe. The right panel of Figure 16 shows the combination of the likelihood contours obtained with three different observational approaches: i) type-Ia $\mathrm{SNe}$ (Tonry et al. 2003; Riess et al. 2004); ii) CMB (Spergel et al. 2003; Bennett et al. 2013); iii) galaxy clusters (Schuecker et al. 2003; Schuecker 2005). One can see that the cosmic matter density is close to $\Omega_{m}=0.3$, and that the normalized cosmological constant is around $\Omega_{\Lambda}=0.7$. This sums up to unit total cosmic energy density and suggests a spatially flat universe. However, the density of cosmic matter growths with redshift like $(1+\mathrm{z})^{3}$ whereas the density $\rho_{\Lambda}$ related to the cosmological constant $\Lambda$ is independent of $\mathrm{z}$. The final results from WMAP
(Bennett et al. 2013) shows a little misalignment with the line of "flat Universe". Thus it is necessary to be careful in the conclusions.

\subsection{Hubble Constant}

The Hubble constant $\left(\mathrm{H}_{0}\right)$ is one of the most important numbers in cosmology because it is needed to estimate the size and age of the universe. The important problem of determination of $\mathrm{H}_{0}$ value is one of the most exciting. Indeed, in the literature it is possible to find many determinations coming from different experiments using different methods. However, it is very complicated to obtain a true value for $\mathrm{H}_{0}$. It is necessary to have two measurements: i) spectroscopic observations that reveal the galaxy's redshift, indicating its radial velocity; ii) the galaxy's precise distance from Earth (and this is the most difficult value to determine).

For a discussion about $\mathrm{H}_{0}$ see Giovannelli \& Sabau-Graziati (2014a and the references therein).

However, Riess et al. (2011) with the HST determined a value of $\mathrm{H}_{0}=73.8 \pm 2.4 \mathrm{~km} \mathrm{~s}^{-1} \mathrm{Mpc}^{-1}$. This value agrees with the WMAP results: $\mathrm{H}_{0}=71.0$ $\pm 2.5 \mathrm{~km} \mathrm{~s}^{-1} \mathrm{Mpc}^{-1}$ (Komatsu et al. 2011). Bennett et al. (2014) discussed the progress occurred in recent years for determining the Hubble constant: results coming from the cosmic distance ladder measurements at low redshift and CMB measurements at high redshift. The CMB is used to predict the current expansion rate of the universe by best-fitting cosmological model. At low redshift baryon acoustic oscillation (BAO) measurements have been usedalthough they cannot independently determine $\mathrm{H}_{0}-$ for constraining possible solutions and checks on cosmic consistency. Comparing these measurements they found $\mathrm{H}_{0}=69.6 \pm 0.7 \mathrm{~km} \mathrm{~s}^{-1} \mathrm{Mpc}^{-1}$.

Does this determination, finally, close the history about the search of the "true" value of $\mathrm{H}_{0}$ ?

\subsection{Reionization Epoch}

Ground-based observations of the CMB on subdegree angular scales suggest that the gas content of the universe was mostly neutral since recombination at $\mathrm{z} \sim 1000$ until about $\mathrm{z} \sim 100$ (Gnedin 2000 and references therein) because earlier reionization would have brought the last scattering surface to lower redshift, smoothing the intrinsic CMB anisotropy. At the same time, we know that the universe is highly ionized, since $\mathrm{z} \approx 5$, from observations of the spectra of quasars with the highest redshifts (e.g. Giallongo et al. 1994). This change of the ionization state of the universe from neutral to highly ionized is called 
"reionization". How large is the redshift to which the reionization started and stopped is object of strong debate. In our opinion probably it is possible to put a reasonable limit to the epoch of the reionization end $(z \sim 6)$, looking at the paper by Toshikawa et al. (2012).

An interesting review about The epoch of reionization was published by Zaroubi (2013), and a discussion about this topic can be found in Giovannelli \& Sabau-Graziati (2016a and the references therein).

\subsubsection{Extragalactic Background Light}

The intergalactic space is filled with the light produced by all the stars and accreting compact objects that populated the observable Universe throughout the whole cosmic history. This relic cosmic background from IR to UV is called the diffuse Extragalactic Background Light (EBL), long before known as DEBRA (Ressel \& Turner 1990).

Direct measurements of the EBL are difficult due to bright local foregrounds. A powerful approach for probing these diffuse radiation fields in the UV to far-IR bands is through $\gamma-\gamma$ absorption of highenergy photons. Actually pair production $\left(\mathrm{e}^{+} \mathrm{e}^{-}\right)$ against EBL photons with wavelengths from ultraviolet to infrared is effective at attenuating $\gamma$-rays with energy above $\sim 10 \mathrm{GeV}$. This process introduces an attenuation in the spectra of $\gamma$-ray sources above a critical energy (e.g. Costamante 2012; Buson 2014).

The last decade has been foreboding of a full coverage of the HE-VHE $\gamma$-ray energy band, thanks to the many ground- and space-based high sensitivity experiments. Thus it has been possible to collect a large amount of data from many extragalactic emitters at high redshift (e.g. Costamante 2012). Thanks to measurements of the quasar 3C 279 (z $\simeq 0.54$ ) obtained with the MAGIC experiment (Albert et al. 2008), and with the many sources at high redshift, including Gamma Ray Bursts (GRBs) measured with the FERMI observatory (Abdo et al. 2010), it has been demonstrated that the Universe is more transparent to $\gamma$-rays than before believed (Coppi \& Aharonian 1997).

Cooray (2016) reviews the Extragalactic Background Light Measurements and Applications. This review covers the measurements related to the extragalactic background light intensity from $\gamma$-rays to radio in the electromagnetic spectrum over 20 decades in wavelength. Figure 17 shows such EBL measurements that updated those reported by Ressel \& Turner (1990). It is important to remark that the numerous measurements in the range of the VHE

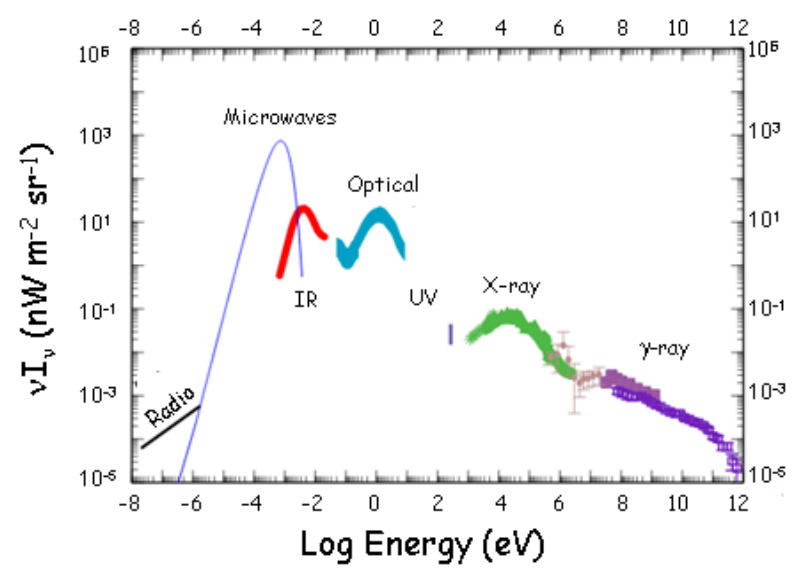

Fig. 17. Intensity of the extragalactic background $\left(\nu \mathrm{I}_{\nu}\right.$ in units of $\mathrm{nW} \mathrm{m}^{-2} \mathrm{sr}^{-1}$ ) as a function of the energy (after Cooray 2016).

$\gamma$-rays $(\log \mathrm{E} \approx 9-13 \mathrm{eV}$ ) have filled the zone where no measurements or only upper limits were available in the 1990-ies.

The CMB remains the best measured spectrum with an accuracy better than 1\%. Durrer (2015) in her interesting review describes the discovery of the cosmic microwave background radiation in 1965 and its impact on cosmology in the 50 years that followed.

Henry et al. (2015) discussed the diffuse cosmic background radiation in the Galaxy Evolution Explorer far-ultraviolet (FUV, 1300-1700 A). They deduced that the UV diffuse cosmic background radiation originates only partially in the dust-scattered radiation of FUV-emitting stars: the source of a substantial fraction of the FUV background radiation remains a mystery. They also discussed about our limited knowledge of the cosmic diffuse background at ultraviolet wavelengths shortward of $\mathrm{Ly} \alpha$ - it could be that a "second component" of the diffuse FUV background persists shortward of the Lyman limit and is the cause of the reionization of the universe.

\subsection{Gamma Ray Bursts}

Long discussions about Gamma-ray bursts (GRBs) can be found in numerous publications. A list of these can be found in GSG2004 and in Giovannelli \& Sabau-Graziati (2016a).

Although big progress has been obtained in the last few years, GRBs theory needs further investigation in the light of the experimental data coming from old and new satellites, often coordinated, such as BeppoSAX or BATSE/RXTE or ASM/RXTE or IPN or HETE or INTEGRAL or SWIFT or AGILE or FERMI or MAXI. Indeed, in spite of thousands 
papers appeared in the literature since the discovery of GRBs, the problem of their energy emission is still elusive: i) what is jet's composition? (kinetic or magnetic?); ii) where is dissipation occurring? (photosphere? deceleration radius?); iii) how is radiation generated? (synchrotron, Inverse Compton, hadronic?) (Zhang 2013a,b).

Kumar \& Zhang (2015) in a review paper discussed what we have learned about relativistic collisionless shocks and particle acceleration from GRB afterglow studies, and the current understanding of radiation mechanism during the prompt emission phase. They pointed out how these explosions may be used to study cosmology, e.g. star formation, metal enrichment, reionization history, as well as the formation of first stars and galaxies in the Universe.

The idea that GRBs could be associated to gravitational waves (GWs) emission is now popular. Indeed, short GRBs are believed to be produced by the mergers of either double NSs or NS-BH binaries (Nakar 2007) and the recent observation of a kilonova associated with GRB130603B (Tanvir et al. 2013; Berger, Fong, \& Chornock 2013) lends support to this hypothesis. In a recent review, D'Avanzo (2015) discussed the observational properties of short GRBs and showed how the study of these properties can be used as a tool to unveil their elusive progenitors and provide information on the nature of the central engine powering the observed emission. The increasing evidence for compact object binary progenitors makes short GRBs one of the most promising sources of gravitational waves for the forthcoming Advanced LIGO/Virgo experiments. This idea obtained recently its experimental verification with the detection of GW 170817 event associated with the GRB 170817A (Abbott et al. 2017a,b).

Thanks to the NASA's Swift satellite we assisted to ten years of amazing discoveries in time domain astronomy. Its primary mission is to chase GRBs. The list of major discoveries in GRBs and other transients includes the long-lived X-ray afterglows and flares from GRBs, the first accurate localization of short GRBs, the discovery of GRBs at high redshift $(z>8)$ (Gehrels \& Cannizzo 2015). And essentially thanks to these discoveries we are now closer to understand the real nature of GRBs.

The recent review by Bernardini (2015) discussed how the newly-born millisecond magnetars can compete with black holes as source of the GRB power, mainly with their rotational energy reservoir. They may be formed both in the core-collapse of massive stars, and in the merger of neutron star or white dwarf binaries, or in the accretion-induced collapse of a white dwarf, being thus a plausible progenitor for long and short GRBs, respectively.

Ghirlanda et al. (2015) discussed about the apparent separation of short and long GRBs in the hardness ratio vs duration plot. This separation has been considered as a direct evidence of the difference between these two populations. The origin of this diversity, however, has been only confirmed with larger GRB samples but not fully understood. They concluded that short and long GRBs have similar luminosities and different energetics (i.e. proportional to the ratio of their average durations). Then, it seems that the results are pointing toward the possibility that short and long GRBs could be produced by different progenitors but the emission mechanism responsible for their prompt emission might be similar.

Piron (2016) in his review discussed the updated knowledge of GRBs at very high energies. Their huge luminosities involve the presence of a newborn stellar-mass black hole emitting a relativistic collimated outflow, which accelerates particles and produces non-thermal emissions from the radio domain to the highest energies. He reviewed recent progresses in the understanding of GRB jet physics above $100 \mathrm{MeV}$, based on Fermi observations of bright GRBs, and discussed the physical implications of these observations and their impact on GRB modeling.

Recently Arnon Dar (2017) proposed again to the attention of the international community his Cannonball (CB) model for explaining the physics of GRBs. In the CB model, GRBs and their afterglows are produced by the interaction of bipolar jets of highly relativistic plasmoids (CBs) of ordinary matter with the radiation and matter along their trajectory. Such jetted CBs are presumably ejected in accretion episodes of fall-back material on the newly formed compact stellar object in core-collapse supernovae (SNe) of Type Ic, in merger of compact stellar objects in close binary systems, and in phase transitions in compact stars (Shaviv \& Dar 1995; Dar 1997; Dar \& De Rujula 2000; Dado \& Dar 2013a). Dado, Dar \& De Rújula (2009) discussed a long series of different SWIFT GRBs, showing that the CB model fits all their broadband light curves. Dado \& Dar (2013b) discussed the jet break in the Xray afterglow of GRBs that appears to be correlated to other properties of the X-ray afterglow and the prompt gamma ray emission, but the correlations are at odds with those predicted by the conical fireball 

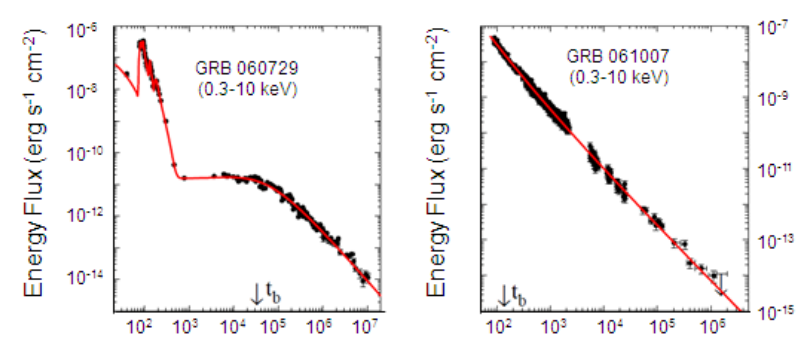

Time since Trigger (s)
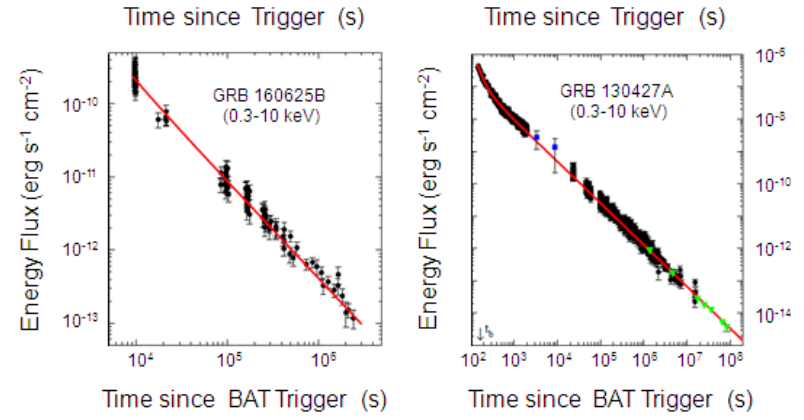

Fig. 18. The 0.3-10keV X-ray light-curve measured with the Swift XRT (Evans et al. 2009), and the comparison between Swift observations and their CB-model description (adapted from Dado \& Dar 2016) for: top left GRB 060729, top right GRB 061007, bottom left GRB 160625B, bottom right GRB 130427A. This latter figure reports data from different experiments: Swift XRT (black circles), XMM Newton and Chandra (green triangles) (De Pasquale et al. 2016), and the two MAXI data points (blue squares) (Maselli et al. 2014) at $t=3257 \mathrm{~s}$ and $\mathrm{t}=8821 \mathrm{~s}$. The BAT trigger time is marked with $t_{b}$.

(FB) model of GRBs (Piran 1999). On the contrary they are in good agreement, however, with those predicted by the CB model of GRBs.

Finally, Dado \& Dar (2016) discussed on the critical test of gamma-ray bursts theories and demonstrated definitively the validity of the CB model against the popular FB model (Piran 1999).

Figure 18 shows, as example, the fits of light curves of GRB 060729, GRB 061007, GRB 160625B, and GRB 130427A by using the CB model.

In our opinion the problem of the models for explaining the behaviour of GRBs can be considered closed. The CB model is the best in absolute for the description of the physics governing the GRBs.

\section{ACCRETION PROCESSES}

Accretion is a universal phenomenon that takes place in the vast majority of astrophysical objects. The progress of ground-based and space-borne observational facilities has resulted in the great amount of information on various accreting astrophysical objects, collected within the last decades. The accre-

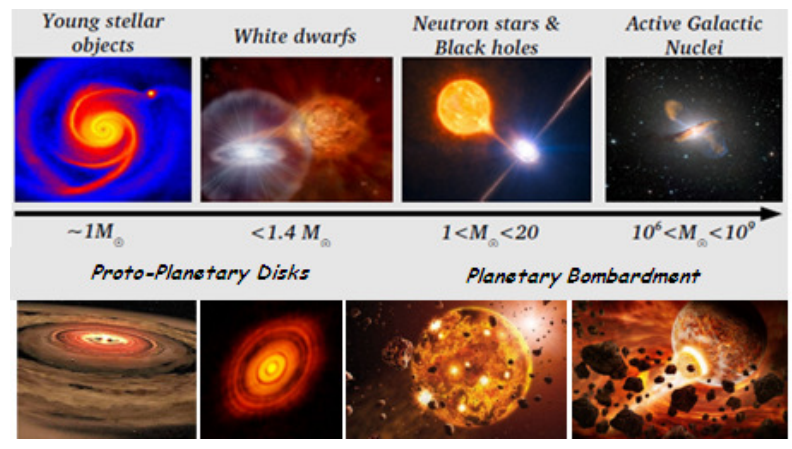

Fig. 19. Accretion processes in different cosmic sources (Giovannelli \& Sabau-Graziati 2016b, after Scaringi 2015).

tion is accompanied by the process of extensive energy release that takes place on the surface of an accreting object and in various gaseous envelopes, accretion disk, jets and other elements of the flow pattern. The results of observations inspired the intensive development of accretion theory, which, in turn, enabled us to study unique properties of accreting objects and physical conditions in the surrounding environment. One of the most interesting outcomes of this intensive study is the fact that accretion processes are, in a sense, self-similar on various spatial scales from planetary systems to galaxies.

This fact gives us new opportunities to investigate objects that, by various reasons, are not available for direct study.

Cataclysmic variable stars are unique natural laboratories where one can conduct the detailed observational study of accretion processes and accretion disks.

Figure 19 shows a sketch of cosmic systems where accretion processes occur (Giovannelli \& SabauGraziati 2016b, after Scaringi 2015).

An international workshop on Accretion Processes in Cosmic Sources: Young Stellar Objects, Cataclysmic Variables and Related Objects, X-ray Binary Systems, Active Galactic Nuclei was organized by us in collaboration with several colleagues of different international institutions, and took place in Saint Petersburg (Russian Federation) on September 2016. The proceedings will discuss in details the physics of accretion processes in all the cosmic sources shown in Figure 19 (Giovannelli \& SabauGraziati 2017).

\section{HABITABLE ZONE IN THE MILKY WAY AND EXOPLANETS}

A deep discussion about this fundamental problem has been published by Giovannelli \& Sabau- 
Graziati (2016a) and references therein. We can briefly summarize in the following.

The most important questions about the possible origin of life in our Universe became a real scientific question in the last couple decades when it appeared a near certainty that other planets must orbit other stars. And yet, it could not be proven, until the early 1990's. Then, radio and optical astronomers detected small changes in stellar emission which revealed the presence of first a few, and now many, planetary systems around other stars. We call these planets "exoplanets" to distinguish them from our own solar system neighbors (http://science.nasa.gov/astrophysics/ focus-areas/exoplanet-exploration/).

The research of potential habitable exoplanets has been strongly supported during last two decades. Indeed, this field of astrophysics is now probably the most exciting since the discovery of planets Earthlike could open a serious debate about the possibility of life outside of our solar system.

The presence of numerous exoplanets in the vicinity of our solar system - within a distance of $\sim 0.8 \mathrm{pc}$ - plays an important role in speculating about the possible number of such exoplanets within the whole habitable zone of our galaxy. Such habitable zone has an internal radius of $\sim 4 \mathrm{kpc}$ and an external radius of $\sim 11 \mathrm{kpc}$, as shown in Figure 20, where the habitable zone in a Milky Way-like galaxy is represented in green. The number of stars contained in this zone is $\approx 10 \%$ of the total number of stars in the Galaxy. Taking into account that the thickness of the disk is $\approx 1 \mathrm{kpc}$, as evaluated by the differential rotation of the Galaxy, the habitable volume is $\sim 330 \mathrm{kpc}^{3}$. Therefore, if in a volume of $\approx 2 \mathrm{pc}^{3}$ there are 808 Earth-like planets detected, in the habitable zone of our Galaxy we could expect $\approx 133 \times 10^{6}$ Earth-like planets. It is evident that the probability of finding numerous habitable planets becomes very high. Next generation instruments ground- and space-based will provide valuable information about this intriguing problem.

A strong support on the possibility of having numerous habitable planets is coming from the discovery of "usual" presence of water in the universe. We knew that all the water found on Earth, has been transported by small bodies such as comets and asteroids. On the contrary, the work "The ancient heritage of water ice in the solar system" (Cleeves et al. 2014) has carried the knowledge one step further. It is understood that the water now present in Earth's oceans, and is present in other solar system bodies, has remained virtually unchanged with re-

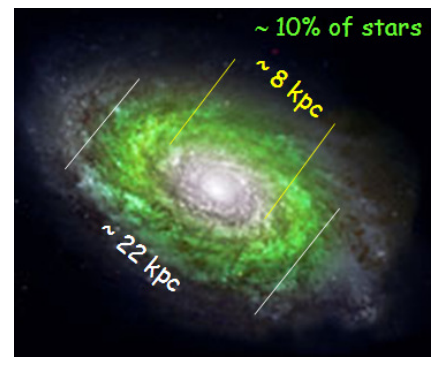

Fig. 20. Habitable zone of a Milky Way-like galaxy (Giovannelli \& Sabau-Graziati 2016a after Lineweaver, Fenner, \& Gibson 2004).

spect to that in the interstellar medium. This means that this water has not changed during the process of planet formation. This allows us to understand that the initial conditions that have favored the emergence of life are not unique, i.e. not dependent on the unique characteristics of our solar system. They can, however, be common in space.

An intriguing question about the probability of finding a number of civilization in the Galaxy arises. It is now evident that Drake's formula (Drake 1962) must be object of a robust revision.

For years, the search for manifestations of extraterrestrial civilizations has been one of humanity's most ambitious projects. Major efforts are now focused on the interception of messages from extraterrestrial civilizations, and the millimeter range is promising for these purposes (Dyson 1960). The Millimetron space observatory is aimed at conducting astronomical observations to probe a broad range of objects in the Universe in the wavelength range $20 \mu \mathrm{m}$ to $20 \mathrm{~mm}$, including the search for extraterrestrial life (Kardashev et al. 2014, and references therein).

\section{CONCLUSIONS}

In this review paper we have discussed several arguments that in our opinion are fundamental for the comprehension of the physics of our Universe. We have emphasized with some examples the use of wisdom in physics. We hope to have given some hints to the readers in order to adopt wisdom during the preparation of their papers.

It is important the Great example of synergy between Astrophysics and History we discussed for demonstrating that Sedov's formula for determining the age of SNRs can be revisited, thanks to the recalibration of the age of the SNR Vela Jr (Aschenbach 2016) experimentally supported by a historical document.

We discussed about the small and big spaceand ground-based experiments that provide mea- 
surements necessary for the advancement of the knowledge of the physics of our Universe. Thanks to these results we discussed the present situation about the problems resolved and those still open, far from the completeness due to our limited knowledge.

A final section about the Habitable Zone in the Milky Way and Exoplanets briefly commented the fundamental problem about the research of life in the Universe. With this new-born field of science we could finally prove that the "Bridge between the Big Bang and Biology" (Giovannelli 2001) is, not only obviously existing, but really can be traveled over.

Finally we can conclude with Figure 21 that clearly explain all the mysteries of our Universe (Giovannelli 2000). People who are able to read this sentence can understand that "The truth is written in the book of the Nature. We must learn to read this book".

$$
\begin{aligned}
& \text { 自然以山教科書には } \\
& \text { 真农がある。 } \\
& \text { ただ我々は } \\
& \text { 諘多方学山゙ }
\end{aligned}
$$

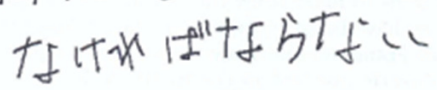

Fig. 21. Understanding our Universe (Giovannelli 2000).

Acknowledgments: Many thanks to Dr. Alberto Javier Castro-Tirado for the invitation and for a partial support to FG for attending this workshop. This research has made use of the NASA's Astrophysics Data System; the NASA Exoplanet Archive, which is operated by the California Institute of Technology, under contract with the National Aeronautics and Space Administration under the Exoplanet Exploration Program.

\section{REFERENCES}

Aad, G. (The ATLAS Collaboration): 2012, PhL B, 718, 369

Aaij, R., et al. 2015, arXiv:1507.03414v2 [hep-ex] 25 Jul. Abbott, B. P., et al. 2016a, PRL, 116, 061102

Abbott, B. P., et al. 2016b, PhRvL, 116, 1103

Abbott, B. P., et al. 2016c, LRR, 19, 1

Abbott, B. P., et al. 2017a, PhRvL, 119, 1101

Abbott, B. P., et al. 2017b, ApJL, 848, 12

Abdo, A. A., et al. 2010, ApJ, 723, 1082

Ade, P. A. R., et al. 2015, PhRvL, 114, 1301
Adelberger, E. G., Austin, S. M., Bahcall, J. N., et al. 1998, RvMP, 70, 1265

Adelberger, E. G., García, A., Robertson, R. G., et al. 2011, RvMP, 83, 195

Anders, M., et al. 2013, EPJA, 49, 28

Anders, M., et al. 2014, PhRvL, 113, 2501

Aschenbach, B. 1998, Natur, 396, 141

Aschenbach, B. 2016, in Frontier Research in Astrophysics II. Online at https://pos.sissa.it/ cgi-bin/reader/conf. cgi? confid=269, id. 36

Bennett, C. L., et al. 2013, ApJS, 208, 20

Bennett, C. L., et al. 2014, ApJ, 794, 135

Berger, E., Fong, W., \& Chornock, R. 2013, ApJL, 774, L23

Bernardini, M. G. 2015, JHEAp, 7, 64

de Bernardis, et al. 2000, Natur, 404, 955

de Bernardis, et al. 2014, Talk at the Mondello Workshop Frontier Research in Astrophysics.

Bisnovatyi-Kogan, G. S. 2002, Black Hole Astrophysics 2002, ed. H. K. Lee \& M. Park (World Scientific Publishing), 187

Bisnovatyi-Kogan, G. S. \& Giovannelli, F. 2017, A\&A, 599, 55

Blandford, R. D. \& Kochanek, C. S. 2004, in Dark Matter in the Universe, ed. J. Bahcall, T. Piran, \& S. Weinberg (London, UK: World Scientific Publishing), 103

Bloom, J., Castro-Tirado, A. J., Hanlon, L., \& Kotani, T. 2010, I Workshop on Robotic Autonomous Observatories, AdAst, 2010

Bouvier, J. 1990, AJ, 99, 946

Bucher, M. (on behalf of the PLANCK Collaboration): 2015, NPPP, 267, 245

Buckley, D. 2015, talk at the Palermo Workshop on The Golden Age of Cataclysmic Variables and Related Objects - III

Buson, S. 2014, in Frontier Research in Astrophysics: Old and New Results, ed. F. Giovannelli \& L. Sabau-Graziati, http://pos.sissa.it/cgi-bin/ reader/conf . cgi? confid= $\backslash \backslash 237$

Caballero-García, M. D., Pandey, S. B., Hiriart, D., \& Castro-Tirado, A. J. 2016, RMxAC, 48, 83

Cardini, A. 2015, Interview of Alessandro Cardini, P.I. of LHCb, 14th July 2015

Castro Cerón, J. M. 2013, Frascati Workshop on Multifrequency Behaviour of High Energy Cosmic Sources, ed. F. Giovannelli \& L. S. Graziati

Castro-Tirado, A. J. 2008, NEA, 3, 131

Castro-Tirado, A. J. 2010a, AdAst, 2010, 60

Castro-Tirado, A. J. 2010b, AdAst, 2010, 68

Cleeves, L. Ilsedore, et al. 2014, Sci, 345, 1590

Cooray, A. 2016, RSOS, 350555

Coppi, P. S. \& Aharonian, F. A. 1997, ApJ, 487, L9

Costamante, L. 2012, in Multifrequency Behaviour of High Energy Cosmic Sources, ed. F. Giovannelli \& L. Sabau-Graziati, MmSAI, 83, 138

Crites, A. T., et al. 2015, ApJ, 805, 36

Dado, S., Dar, A., \& De Rújula, A. 2009, ApJ, 696, 994

Dado, S. \& Dar, A. 2013a, ApJ, 775, 16 
Dado, S. \& Dar, A. 2013b, A\&A, 558, 115

Dado, S. \& Dar, A. 2016, PhRvD, 94, 3007

Dai, X. \& Kochanek, C. S. 2005, ApJ, 625, 633

Dar, A. 1997, in Very High Energy Phenomena in the Universe, ed. Y. Giraud-Heraud \& J. T. T. Van (Editions Frontieres), 69

Dar, A. 2017, talk at the Frascati Workshop 2017, Multifrequency Behaviour of High Energy Cosmic Sources - XIII

Dar, A. \& De Rújula, A. 2000, arXiv: 0008474

D'Avanzo, P. 2015, JHEAp, 7, 73

De Angelis, A., et al. 2017, ExA, 44, 25

De Pasquale, M., Page, M. J., Kann, D. A., et al. 2016, MNRAS, 462, 1111

Di Leva, A., et al. 2014, PhRv C, 89, 5803; and erratum PhRv C, 90, 9902

Drake, F. D. 1962, Intelligent Life in Space, (New York, NY: Macmillan)

Durrer, R. 2015, CQGra, 32, 124007

Dyson, F. 1960, Sci, 131, 667

Erickson, J. K., Gratton, S., Steinhardt, P. J., \& Turok, N. 2007, PhRvD, 75, 123507

Evans, P. A., et al. 2009, MNRAS, 397, 1177

Flauger, R., Hill, J. C., \& Spergel, D. N. 2014, JCAP, 08,39

Gehrels, N. \& Cannizzo, J. K. 2015, JHEAp, 7, 2

Gell-Mann, M. 1964, PhL, 8, 214

Ghirlanda, G., Bernardini, M. G., Calderone, G., \& D'Avanzo, P. 2015, JHEAp, 7, 81

Giallongo, E., et al. 1994, ApJ, 425, L1

Gianotti, F. 2012, 4th July, talk at CERN

Gilmozzi, R. \& Spyromilio, J. 2007, Msngr, 127, 11

Giovannelli, F. 1994, SSRv, 69, 1

Giovannelli, F. 2000, in The Evolution of The Milky Way: Stars versus clusters, ed. F. Matteucci \& F. Giovannelli (Dordrecht: Kluwer Academic Publishers), 619

Giovannelli, F. 2010, in Multifrequency Behaviour of High Energy Cosmic Sources, ed. F. Giovannelli \& L. Sabau-Graziati, MmSAI, 81, 510

Giovannelli, F. 2013, AcPol, 53, 483

Giovannelli, F. 2016, in High Energy Astrophysics in Southern Africa, heas.confE, (Southern Africa: HEASA), Online at http://pos.sissa.it/cgi-bin/ reader/conf . cgi? confid=275, id. 31

Giovannelli, F., Bisnovatyi-Kogan, G. S., Bruni, I., et al. 2015, AcA, 65, 107

Giovannelli, F., Bisnovatyi-Kogan, G. S., \& Klepnev, A. S. 2013, A\&A, 560, A1

Giovannelli, F., La Padula, C. D., Sabau-Graziati, L., et al. 1999b, in Frontier Objects in Astrophysics and Particle Physics, ed. F. Giovannelli \& G. Mannocchi, SIF, 65, 623

Giovannelli, F. \& Sabau-Graziati, L. 2001, The bridge between the big bang and biology: stars, planetary systems, atmospheres, volcanoes: their link to life, ed. F. Giovannelli (Rome, Italy: Consiglio Nazionalle delle Ricerche)

Giovannelli, F., Sabau Graziati, L., La Padula, C., et al.
1993, A\&AS, 97, 395

Giovannelli, F., Sabau-Graziati, L., La Padula, C. D., et al. 1999a, MmSAI, 70, 1317

Giovannelli, F., Sabau-Graziati, L., Isern, J., 2001, in Frontier Objects in Astrophysics and Particle Physics, ed. F. Giovannelli \& G. Mannocchi, SIF, 73, 565

Giovannelli, F., Sabau-Graziati, L., Isern, J., et al. 2002a, SIXE (Spanish Italian X-ray Experiment) - Feasibility Study Report. (PNIE-CICYT Report (ESP971784-E grant), IASF, Technical Report No. 4, December 2002

Giovannelli, F., Sabau-Graziati, L., Isern, J., et al. 2002b, MmSAI, 73, 402

Giovannelli, F. \& Sabau-Graziati, L. 2004, SSR, 112, 1

Giovannelli, F. \& Sabau-Graziati, L. 2011, AcPol, 51, 21

Giovannelli, F. \& Sabau-Graziati, L. 2012a, MnSAI, 83, 17

Giovannelli F. \& Sabau-Graziati, L. 2014a, in Multifrequency Behaviour of High Energy Cosmic Sources, 2014,1

Giovannelli, F. \& Sabau-Graziati, L. 2014b, in Frontier Research in Astrophysics, 2014, 1

Giovannelli, F. \& Sabau-Graziati, L. 2015, in Multifrequency Behaviour of High Energy Cosmic Sources, 2015, 1

Giovannelli, F. \& Sabau-Graziati, L. 2016a, in Frontier Research in Astrophysics II, 2016, 1

Giovannelli, F. \& Sabau-Graziati, L. 2016b, RMxAC, 48, 1

Giovannelli, F. \& Sabau-Graziati, L. 2017, Accretion Processes in Cosmic Sources, 1

Giovannelli, F., Vittone, A. A., Rossi, C., et al. 1995, A\&AS, 114, 341

Gnedin, N. Y. 2000, ApJ, 535, 530

Gustavino, C. 2007, in Frontier Objects in Astrophysics and Particle Physics, ed. F. Giovannelli \& G. Mannocchi, (Bologna, Italy: Compositori), 93, 191

Gustavino, C. 2009, in Frontier Objects in Astrophysics and Particle Physics, ed. F. Giovannelli \& G. Mannocchi, (Bologna, Italy: Compositori), 98, 77

Gustavino, C. 2011, in Frontier Objects in Astrophysics and Particle Physics, ed. F. Giovannelli \& G. Mannocchi, (Bologna, Italy: Compositori), 103, 657

Gustavino, C. 2012, in Nuclei in the Cosmos (NIC XII), http://pos.sissa.it/cgi-bin/reader/conf. cgi? confid=146, id.74

Gustavino, C. 2013, AcPol, 53, 534

Guziy, S., Pandey, S. B., Tello, J. C., \& Castro-Tirado, A. J. (ed.). 2012, II Workshop on Robotic Autonomous Observatories, ASInC, 7

Hamuy, M., et al. 2012, MmSAI, 83, 388

Haridasu, B. S., Lukovic, V. V., D’Agostino, R., \& Vittorio, N. 2017, A\&A, 600, 1

Hartwig, T., Volonteri, M., Bromm, V., et al. 2016, MNRAS, 460, 74

Henry, R. C., Murthy, J., Overduin, J., \& Tyler, J. 2015, ApJ, 798, 14 
Hessman, F. V. 2001a, ASPC 246, 13

Hessman, F. V. 2001b, ASPC 246, 357

Hudec, R. 2017, Multifrequency Behavior of High Energy Cosmic Sources - XII

Ijjas, A., Steinhardt, P. J., \& Loeb, A. 2013, PhLB, 723, 261

Ijjas, A. 2016, arXiv161002752

Incandela, J. 2012, UCSB/CERN, Talk on July 4, 2012

Isern, J., Giovannelli, F. (Principal Investigators), Angúlo, M., et al. 1999a, SIXE (Spanish Italian Xray Experiment) - Feasibility Study Report, PNIECICYT Report (ESP97-1784-E grant)

Isern, J., Bravo, E., Gómez-Gomar, J., et al. 1999b, AIPC, 499, 110

Jakobs, K. \& Seez, C. 2015, SchpJ, 10, 32413

Kardashev, N. S., et al. 2014, PhyU, 57, 1199

Keating, Brian G., et al. 2003, SPIE, 4843, 284

Kochanek, C. S. 2003, ASPC, 291, 245

Komatsu, E., et al. 2011, ApJS, 192, 18

Kumar, P. \& Zhang, B. 2015, PhR, 561, 1

Lamzin, S. A., Bisnovatyi-Kogan, G. S., Errico, L., et al. 1996, A\&A 306, 877

Lasota, J.-P. 2001, NewAR, 45, 449

Lineweaver, C. H., Fenner, Y., \& Gibson, B. K. 2004, Sci, 303, 59

Lipunov, V. M. 1987, Ap\&SS, 132, 1

Lipunov, V. M. \& Postnov, K. A. 1988, Ap\&SS, 145, 1

Lipunov, V. M., Nazin, S. N., Panchenko, I. E., Postnov, K. A., \& Prokhorov, M. E. 1995, A\&A, 298, 677

Lipunov, V., et al. 2010, AdAst, 2010, 30

Luisi, P. L. \& Capra, F. 2014, The Systems View of Life: A Unyfying Vision, (Cambridge, MA: CUP)

Maselli, A., Melandri, A., Nava, L., et al. 2014, Sci, 343, 48

Meylan, G., Jetzer, P., \& North, P. 2006, Gravitational lensing: strong, weak and micro, ed. G. Meylan, P. Jetzer, \& P. North (Berlin: Springer)

Nakar, E. 2007, PhR, 442, 166

Nielsen, J. T., Guffanti, A., \& Sarkar, S. 2016, NatSR, 6, 35596

Ogburn, R. W., IV et al. 2010, SPIE, 7741, 1

Orosz, J. A., Remillard, R. A., Bailyn, C. D., \& McClintock, J. E. 1997, ApJL, 478, L83

Perlmutter, S., Aldering, G., Goldhaber, G., et al. 1999, ApJ, 517, 565

Pignata, G., et al. 2009, AIPC, 1111, 551

Piran, T. 1999, PhR, 314, 575

Piron, F. 2016, RPhy, 17, 617

Planck Collaboration 2014, A\&A, 571, 31

Rees, M. J. 1988, in Origins, ed. A. C. Fabian (Cambridge, MA: CUP), 1

Reig, P., Fabregat, J., \& Coe, M. J. 1997, A\&A, 322, 193

Ressel, M. T. \& Turner, M. S. 1990, ComAp, 14, 323

Riess, A. G., Filippenko, A. V., Challis, P., et al. 1998, AJ, 116, 1009

Riess, A. G., et al. 2004, ApJ, 607, 665

Riess, A. G., et al. 2011, ApJ, 730, 119

Rix, H-W. \& Bovy, J. 2013, A\&ARv, 21, 61
Scaringi, S. 2015, Talk at the Palermo Workshop on The Golden Age of CVs and Related Objects III

Schuecker, P. 2005, RvMA, 18, 76

Schuecker, P., et al. 2003, A\&A, 402, 53

Scott, D. A., et al. 2012, PhRvL, 109, 2501

Shahbaz, T., Bandyopadhyay, R. M., Charles, P. A., et al. 1998, MNRAS, 300, 1035

Shakura, N. I. \& Sunyaev, R. A. 1973, A\&A, 24, 337

Shandarin, S., Habib, S., \& Heitmann, K. 2010, PhRvD, 81,3006

Shaviv, N. J. \& Dar, A. 1995, MNRAS, 277, 287

Shimokawabe, T., et al. 2009, AIPC, 1133, 79

Spergel, D. N., et al. 2003, ApJS, 148, 175

Smak, J. 1984, PASP, 96, 5

Steinhardt, P. J., Turok, N., \& Starkman, G. D. 2008, PhT, 61, 59

Straniero, O., et al. 2013, ApJ, 763, 100

Strieder, F., et al. 2012, PhLB, 707, 60

Tanvir, N. R., Fox, D. B., Levan, A. J., et al. 2009, Natur, 461,1254

Tanvir, N. R., Levan, A. J., Fruchter, A. S., et al. 2013, Natur, 500, 547

Tello, J. C., Riva, A., Hiriart, D., \& Castro-Tirado, A. J. 2014, RMxAC, 45

The CMS Collaboration. 2012a, Sci, 338, 21

The CMS Collaboration. 2012b, PhLB, 716, 30

Tonry, J. L., et al. 2003, ApJ, 594, 1

Topchiev, N. P., et al. 2016a, JPhCS, 675, 2009

Topchiev, N. P., et al. 2016b, JPhCS, 675, 2010

Topchiev, N. P. et al. 2017, JPhCS, 798, 2011

Toshikawa, J., Kashikawa, N., Ota, K., et al. 2012, ApJ, 750,137

Turner, M. S. \& Riess, A. G. 2002, ApJ, 569, 18

Tyson, J. A. 2000, Encyclopedia of Astronomy and Astrophysics, ed. Paul Murdin, article 2144

Tyson, J. A., Kochanski, G. P., \& Dell'Antonio, I. P. 1998, ApJL, 498, L107

Villada, M., Rossi, C., Polcaro, V. F., \& Giovannelli, F. 1999, A\&A, 344, 211

Wheatley, P. J., Mauche, C. W., \& Mattei, J. A. 2003, MNRAS, 345, 49

Williams, L. L. R. \& Schechter, P. L. 1997, A\&G, 38, 10

Winn, J. N., Rusin, D., \& Kochanek, C. S. 2004, Natur, 427,613

Winstein, B. 2007, IJMPD, 16, 2563

Winstein, B. 2009, in From Quantum to Cosmos: Fundamental Physics Research in Space, ed. T. Slava (World Scientific Publishing), 697

Wittman, D. M., Tyson, J. A., Kirkman, D., Dell'Antonio, I., \& Bernstein, G. 2000, Natur, 405, 143

Wolschin, G. 2003, in Time, Quantum and Information, ed. L. Castell \& O. Ischebeck, (Berlin, Germany: Springer), 115

Zaroubi, S. 2013, in The First Galaxies, ASSL, 396, 45

de Zeeuw, T., Tamai, R., \& Liske, J. 2014, Msngr, 158, 3

Zhang, B. 2013a, Talk at the Multi-Messenger Transient 
Workshop, KIAA, China

Zhang, B. 2013b, in Gamma-ray Bursts: 15 Years of GRB

Afterglows-Progenitors, Environments and
Host Galaxies from the Nearby to Early Universe, ed. A. J. Castro-Tirado, J. Gorosabel, and I. H. Park (EAS Publication Series 61), 285 